\title{
Locally Refined Multigrid Solution of the All-Electron Kohn-Sham Equation
}

\author{
Or Cohen, ${ }^{*, \dagger}, \ddagger$ Leeor Kronik, ${ }^{* \dagger}$ and Achi Brandt $*, \mathbb{I}$ \\ Department of Materials and Interfaces, Department of Physics of Complex Systems, and \\ Department of Applied Mathematics and Computer Science, Weizmann Institute of Science, \\ Rehovot 76100, Israel \\ E-mail: or.cohen@weizmann.ac.il; leeor.kronik@weizmann.ac.il; achi.brandt@weizmann.ac.il
}

\begin{abstract}
We present a fully-numerical multigrid approach for solving the all-electron Kohn-Sham equation in molecules. The equation is represented on a hierarchy of cartesian grids, from coarse ones that span the entire molecule to very fine ones that describe only a small volume around each atom. This approach is adaptable to any type of geometry. We demonstrate it for a variety of small molecules and obtain high accuracy agreement with results obtained previously for diatomic molecules using a prolate-spheroidal grid. We provide a detailed presentation of the numerical methodology and discuss possible extensions of this approach.
\end{abstract}

\section{Introduction}

Density functional theory (DFT) is a commonly used approach for first-principles electronicstructure calculations. ${ }^{1,2}$ In DFT, the original Schrödinger equation of the interacting-electron

\footnotetext{
*To whom correspondence should be addressed

${ }^{\dagger}$ Department of Materials and Interfaces

†Department of Physics of Complex Systems

IDepartment of Applied Mathematics and Computer Science, Weizmann Institute of Science, Rehovot 76100, Israel
} 
problem is mapped onto the Kohn-Sham equation of noninteracting electrons, given in atomic units by

$$
\left(-\frac{\nabla^{2}}{2}+V_{i o n}(\mathbf{r})+V_{H}[\rho ; \mathbf{r}]+V_{x c}[\rho ; \mathbf{r}]\right) \varphi_{k}(\mathbf{r})=\varepsilon_{k} \varphi_{k}(\mathbf{r})
$$

Here $\varphi_{k}(\mathbf{r})$ and $\varepsilon_{k}$ are the Kohn-Sham orbitals and eigenvalues, respectively. The tradeoff in this transformation is that in addition to the ionic potential, $V_{i o n}$, which is fixed within the BornOppenheimer approximation, the Kohn-Sham Hamiltonian contains the Hartree potential, $V_{H}$, and the exchange-correlation potential, $V_{x c}$, which are functionals of the electron-density. The latter is defined as

$$
\rho(\mathbf{r})=\sum_{k} f_{k}\left|\varphi_{k}(\mathbf{r})\right|^{2}
$$

where $f_{k}$ are the orbital occupation numbers. The two potentials, $V_{H}$ and $V_{x c}$, account for the classical (Coulomb) and nonclassical electron-electron interaction, respectively.

The ionic potential, $V_{i o n}$, diverges at the atomic positions, causing the orbitals to exhibit strong spatial oscillations. The typical length-scale of these oscillations is $a_{0} / Z$, where $a_{0} \simeq 0.53 \AA$ is the Bohr radius and $Z$ is the ionic charge. In the inter-atomic regions, however, where all the potentials are smooth, the orbitals vary slowly with a typical length-scale of the size of the molecule, which can reach tens of $\AA$ even in simple molecules. These two different length-scales imply that, in the absence of any additional approximations, a uniform real-space discretization of the Kohn-Sham equation would require spanning a large region of space with a relatively fine grid. Similarly, a plane-wave discretization would require a large Fourier basis. Using such naive discretizations in order to obtain the accuracy needed for chemical interpretations would typically consume an inaccessible amount of computational power even for relatively simple molecule.

Dual-scale problems of the type described above are common in many areas of physics and are unavoidable in chemistry. They are often solved by modeling the behaviour of the system on the smaller scale. In DFT this is often done by replacing the singular ionic potential with a pseudopotential, which approximates the screened potential 'felt' by the valence electrons. ${ }^{3,4}$ The use of pseudopotentials yields valence orbitals that are smooth throughout the domain, allowing one to 
obtain the desired accuracy with a relatively small number of grid points or plane waves. In addition, 'burying' the core electrons in the pseudopotential reduces significantly the number of orbitals that one has to compute. Another common approach to this issue is to discretize the wavevectors using an atomic basis set, typically chosen to be a set of gaussians centered around each atom. ${ }^{5}$ These basis sets provide the necessary fine description of the core regions, but suffer from other problems such as basis-incompleteness (see, e.g., section 6 in Cramer $^{5}$ ). In general, pseudopotentials and atomic basis sets induce discretization errors that cannot be made arbitrarily small, but are often smaller than the errors resulting from the approximate form of the exchange-correlation potential, $V_{x c}$. These approximations therefore allow one to carry out sufficiently accurate DFT calculations of large molecules, and are thus an essential ingredient in the success of DFT. Nevertheless, there are certainly cases where by comparison to fully-numerical methods the accuracy of the pseudopotentials and atomic basis sets has been called into question. ${ }^{6,7}$

In recent years, as the DFT approach is being pushed to higher levels of accuracy, the corediscretization errors described above have become the focus of many studies. One example is the application and development of orbital-dependent exchange correlation potentials, for which the construction of pseudopotentials is more difficult. ${ }^{7}$ Another example is the calculation of excited states within the GW approximation, which can be sensitive to the approximation of the core (see, e.g., Gómez-Abalo et al. ${ }^{8}$ and references therein). Such studies and others can therefore benefit from the ability to perform benchmark all-electron calculations (that include both the valence and core orbitals) with arbitrarily accurate discretization of the core.

The real-space finite-difference approach stands out as a promising direction for achieving this goal ${ }^{1}$. Previous real-space solutions of the all-electron Kohn-Sham equation have followed two main approaches. In the first approach, known as grid curving, the grid spacing is taken to be a polynomial function of the coordinates. The two main applications of this idea were based on a prolate-spheroidal grid ${ }^{16,17}$ and on a more general curvilinear grid. ${ }^{18}$ While the prolate-spheroidal

\footnotetext{
${ }^{1}$ An alternative approach for solving the Kohn-Sham equation is the finite-element method. Although many application of this approach involve pseudopotentials (see, e.g., Ref. ${ }^{9-13}$ ), there has been recent progress in the solution of the all-electron problem, achieved using a hierarchy of finite-element bases. ${ }^{14,15}$
} 
grid is limited by construction to diatomic molecules, the curvilinear grid may in principle be used for more complicated geometries. However, there are some substantial difficulties that may arise in the process, as discussed by Beck. ${ }^{19}$

The second approach, employed in the present work, uses a set of grids with different mesh spacing, which form a multigrid. ${ }^{20-22}$ In standard multigrid applications, all the grids span the same region of space. In that case, the equations are in fact fully-discretized on the finest grid level and the purpose of the coarser grid levels is only to accelerate the convergence of the iterative solution method employed on the finest grid. In the present study we use a generalization of this approach, known as local mesh refinement multigrid, whereby several fine grid levels span only a small portion of the entire domain. ${ }^{23,24}$ The coarser grids are then used both to accelerate the iterative solution method and to discretize the equations in regions where only a coarse discretization is needed. In our case, we use a set of cartesian grids that range from very fine grids that span a small region around each nucleus, to very coarse grids that span the entire molecule. This allows one to solve the all-electron Kohn-Sham equation in a general geometry, with a computational cost that depends weakly on the interatomic distances. Another important advantage of this approach over the idea of grid curving is the use of cartesian grids, which enables the use of standard stencils for the discrete derivative and integral operators.

Prior to the present work, multigrid methods have been used for real-space DFT calculations mainly in conjugation with pseudopotentials (see, e.g., Ref. ${ }^{25-31}$ and Beck ${ }^{19}$ for an overview). Because the pseudopotentials yield smooth orbitals, these studies used either very few refinement grids, or none at all. The all-electron Kohn-Sham equation has only been solved in the past using standard multigrid techniques, without employing local mesh refinement. ${ }^{32}$ The use of a large set of locally refined grid levels, needed for obtaining high accuracy with linear scalability of the algorithm in the number of atoms, leads to several challenges that have not been addressed in previous work, as discussed below.

This paper is organized as follows. Section 2.1 is a brief introduction to the standard multigrid approach, provided here for completeness. In Sections 2.2-2.6 we describe the various multigrid 
techniques required specifically in order to tackle a nonlinear eigenproblem in the presence of a singular potential. Notably, we present in Section 2.7 a modified Rayleigh-Ritz procedure, devised for the present study. In Section 3 we present high accuracy results of the Kohn-Sham total energies and eigenvalues obtained for small molecules, such as $\mathrm{H}_{2} \mathrm{O}, \mathrm{CO}_{2}, \mathrm{C}_{6} \mathrm{H}_{6}, \mathrm{CH}_{3} \mathrm{COOH}$, and several diatomic molecules. The results agree with previous fully-numerical studies of diatomic molecules to all digits that are significant, and with calculations based on very large gaussian basis sets to an

accuracy of $10^{-5}$ Hartree. In Section 5 we discuss the scalability of our approach and mention possible extensions which may increase its efficiency.

\section{Numerical Approach}

In this section, we review the numerical methods employed in the present work. For completeness, we begin with a brief introduction to the multigrid approach in Section 2.1. In Section 2.2, we describe the discretization of the Kohn-Sham equation on a set of locally refined grids. At each grid level, the equation is solved using a combination of two relaxation methods, described in Section 2.3. The dependence of $V_{x c}$ and $V_{H}$ on the electron density, $\rho$, implies that the Kohn-Sham equation is in fact a set of nonlinear equations. In Section 2.4, we describe how the iterative nature of the multigrid approach allows one to solve this set of equations using an efficient self-consistency cycle, where all components of the nonlinear system are solved at each grid level. In Section 2.5 we present an algorithm, which, by gradual refinement of the grids, increases the efficiency of the solver and measures the discretization errors. The first step in this algorithm, described in Section 2.6, is a continuation process which yield an initial approximation of the molecular orbitals from the known hydrogen-like wavefunctions. Another ingredient of the self-consistent cycle is the calculation of the Kohn-Sham eigenvalues using the Rayleigh-Ritz procedure, discussed in Section 2.1. When using the standard Rayleigh-Ritz procedure the cycle could not fully converge. This issue, which is in fact due to the local-mesh refinement, was solved by using a modified Rayleigh-Ritz procedure, presented in Section 2.7. 


\subsection{Multigrid Solution of Eigenvalue Problems}

This section provides a brief review of the multigrid approach. Further details can be found in various books published on the topic. ${ }^{20-22}$ We focus here on a formulation known as the full approximation scheme (FAS), which is suitable for nonlinear problems. For simplicity, however, we discuss in this section a general linear equation of the form,

$$
A^{h} \mathbf{u}^{h}=\mathbf{b}^{h}
$$

where $A$ and $\mathbf{b}$ are given while $\mathbf{u}$ is the vector which we seek. The nonlinear case, where $A$ is a function of $\mathbf{u}$, is discussed in Section 2.4 in the context of the Kohn-Sham equation. The superscripts denote in Eq. (3) and throughout this paper the discretization of a vector or a matrix on a grid of mesh spacing $h$. In the case of the Kohn-Sham equation (1), for every Kohn-Sham orbital we have an equation of this type with $\mathbf{u}^{h}$ denoting the wavevector and $\mathbf{b}=0$. In this case, the matrix $A^{h}$ contains contributions from the potentials and the eigenvalue in the diagonal terms and from the finite difference representation of the Laplace operator ${ }^{33}$ in several layers around the diagonal.

The resulting sparsity and diagonal-dominance of the discrete operator of Kohn-Sham equation allows one to solve it using an iterative relaxation procedure. In the present work we use the GaussSeidel (GS) method, which is commonly used in real-space multigrid implementations. ${ }^{20}$ In the GS method the new approximation vector, $\mathbf{u}^{h,(k+1)}$, is given by

$$
u_{i}^{h,(k+1)}=\frac{1}{A_{i i}^{h}}\left(b_{i}^{h}-\sum_{j<i} A_{i j}^{h} u_{j}^{h,(k+1)}-\sum_{j>i} A_{i j}^{h} u_{j}^{h,(k)}\right),
$$

where $\mathbf{u}^{h(k)}$ is the current approximation vector. Each GS sweep consists of applying Eq. (4) sequentially to all the grid points, $i=1, \ldots, L$. In general, the Gauss-Seidel method and related procedures are known to converge for positive definite discrete operators, $A^{h}$, as discussed for instance by Brandt. ${ }^{34}$ However, because they update only a local portion of the approximation 
vector at each iteration, these methods converge slowly when the algebraic error (the difference between $\mathbf{u}^{h,(k)}$ and the solution of the discrete equation) is composed of wave components whose spatial wavelength is large in comparison with the mesh spacing. The multigrid approach has been introduced in order to overcome this slowness by transferring the equation to a set of coarser grids, where the spatial wavelength of the error is comparable to the grid spacing.

The basic unit of the multigrid process is the V-cycle. This cycle iterates from the finest grid level to the coarsest one and back to the finest grid level, with relaxation sweeps employed in each grid level. Throughout this work we assume that the mesh spacing changes between each grid level by a factor of 2, as done in many multigrid implementations. After several relaxation on the finest grid level, where the cycle begins, the approximation vector, $\mathbf{u}^{h,(k)}$, is transferred to a coarser grid level through

$$
\mathbf{u}^{2 h,(k)}=I_{h}^{2 h} \mathbf{u}^{h,(k)}
$$

where $I_{h}^{2 h}$ denotes a grid-transfer operator. The specific form of the transfer operator employed in this work is discussed in Section 2.2. It is important to note that if $\mathbf{u}^{h,(k)}$ is the true solution of $A^{h} \mathbf{u}^{h}=\mathbf{b}^{h}$, the vector $\mathbf{u}^{2 h,(k)}$ defined above will generally not be a solution of $A^{2 h} \mathbf{u}^{2 h}=\mathbf{b}^{2 h}$. The coarse equation should therefore be modified in order to assure that the relaxation on the coarse grid level converges to the coarse version of the fine solution. This is done by adding a defect-correction vector, $\tau^{2 h}$, to the RHS of the coarse equation,

$$
f A^{2 h} \mathbf{u}^{2 h}=\mathbf{b}^{2 h}+\tau^{2 h}
$$

where $\tau^{2 h}$ is defined as

$$
\tau^{2 h}=\left(A^{2 h} \mathbf{u}^{2 h,(k)}-\mathbf{b}^{2 h}\right)-\hat{I}_{h}^{2 h}\left(A^{h} \mathbf{u}^{h,(k)}-\mathbf{b}^{h}\right)+\tau^{h},
$$

and transferred along with the approximation vector. The operator $\hat{I}_{h}^{2 h}$ may in principle be of a different order than that of $I_{h}^{2 h}$ in Eq. (5). The vector $\tau^{h}$ in Eq. (7), denotes the defect correction 
transferred to grid level $h$ from a yet finer grid level. This vector is therefore zero if $h$ is the finest mesh spacing. The addition of $\tau^{h}$ in Eq. (7) is relevant, for example, on grid level $2 h$, where after several relaxation sweeps, we move to grid level $4 h$ and compute $\mathbf{u}^{4 h,(k)}$ and $\tau^{4 h}$.

After several relaxation sweeps on grid level $2 h$ and, if necessary, correction from even coarser grids, we obtain a new coarse approximation vector denoted by $\mathbf{u}^{2 h,(k+q)}$, where $q$ denote the number of changes the vector has undergone. The transfer of these changes back to grid level $h$ is defined as

$$
\mathbf{u}^{h,(k+1)}=\mathbf{u}^{h,(k)}+I_{2 h}^{h}\left(\mathbf{u}^{2 h,(k+q)}-\mathbf{u}^{2 h,(k)}\right),
$$

where $I_{2 h}^{h}$ denotes a coarse-to-fine interpolation operator, often chosen to be the linear interpolation operator.

The typical number of relaxation sweeps necessary on each grid level in each direction of the $V$-cycle is about 2. On the coarsest grid level, however, where we cannot rely on a yet coarser grid level to reduce the slowly converging error components, we have to perform many relaxation sweeps and solve the equation to very low residuals. Because this grid level comprises of relatively few grid points, this additional relaxation, which is sometime even replaced by direct diagonalization, does not require a significant amount of computational work.

Iterating over the $\mathrm{V}$-cycle has been shown to reduce the algebraic error of positive definite equations efficiently, even when the error contains low wavelength components (in comparison to the finest grid spacing). The above formulation of the V-cycle is known as the full approximation scheme (FAS), which is suitable for nonlinear problems, and thus chosen for the present study. Linear problems can be solved using the correction scheme (CS), whereby on each coarse grid level we solve only for the correction to the approximation vector of the finer level, rather than for the approximation vector itself (see section 8.1 in $\operatorname{Brandt}^{20}$ ).

Eigenvalue problems, such as the Kohn-Sham equation, can be solved by employing the GS method for the following equation:

$$
\left(H^{h}-\varepsilon_{m}\right) \varphi_{m}^{h}=0
$$


where $H$ in our case is the Kohn-Sham Hamiltonian, $H=-\frac{\nabla^{2}}{2}+V_{i o n}+V_{H}+V_{x c}$. In general, the eigenvalues are not known, but can be estimated by performing at the end of each V-cycle a Rayleigh-Ritz procedure. ${ }^{35}$ Starting from the current approximation for the set of eigenvalues, $\left\{\varepsilon_{m}^{(k+1)}\right\}$, and eigenvectors, $\left\{\varphi_{m}^{h,(k)}\right\}$, the Rayleigh-Ritz process yields new eigenvalues, $\left\{\varepsilon_{m}^{(k+1)}\right\}$, and a recombination of the wavevectors, denoted by

$$
\varphi_{m}^{h,(k+1)}=\sum_{n=1}^{N} R_{m n} \varphi_{n}^{h,(k+1)}
$$

where $R$ is the recombination matrix and $N$ is the number of eigenvectors. Before describing the Rayleigh-Ritz procedure, we discuss its purpose by examining the relaxation of the Eq. (9) with $\varepsilon_{m}=\varepsilon_{m}^{(k)}$. Assuming that $\varepsilon_{m}^{(k)}$ is slightly different than the true eigenvalue of the discrete equation, the relaxation yields a recombination of eigenvectors with eigenvalues that are close to $\varepsilon_{m}^{(k)}$. The aim of the Rayleigh-Ritz process is to recombine the eigenvectors obtained from the relaxation with the different eigenvalues into a new basis, which is as close as possible to the true eigenbasis of the discrete equation. This is achieved by choosing the matrix $R$ and the eigenvalues such that

$$
\left\langle\varphi_{n}^{h,(k+1)}, \varphi_{m}^{h,(k+1)}\right\rangle=0, \quad\left\langle\varphi_{n}^{h,(k+1)},\left(H^{h}-\varepsilon_{m}^{(k+1)}\right) \varphi_{m}^{h,(k+1)}\right\rangle=0,
$$

where $h$ denotes here is the finest grid spacing. The inner products here and below are computed by a discrete integration scheme, discussed in Section 2.2. One way to understand Eq. (11) is to consider a case where $\varphi^{h,(k)}$ are given as recombination of the true eigenvectors of the discrete equation. In this case, the above choice of $R$ and $\varepsilon_{m}^{(k+1)}$ would yield these true eigenvectors and eigenvalues.

The first step in the Rayleigh-Ritz procedure is the computation of the following matrices:

$$
U_{n m} \equiv\left\langle\varphi_{m}^{h,(k)}, \varphi_{m}^{h,(k)}\right\rangle, \quad M_{n m} \equiv\left\langle\varphi_{m}^{h,(k)}, H^{h} \varphi_{m}^{h,(k)}\right\rangle
$$

Using $U$ we compute a Gram-Schmidt transformation of the wavevectors, denoted by the matrix 
$\Phi$, such that $\Phi^{T} U \Phi=I$. This implies that setting $R=\Phi$ in Eq. (10) yield an orthonormal basis. We then diagonalize the matrix $M$ is this new basis, where it is given by $M^{\prime}=\Phi^{T} M \Phi$. The result is a diagonal matrix, $D$, and a transformation matrix, $P$, such that $D=P^{T} M^{\prime} P$. The diagonal elements of $D$ set the new eigenvalues, $\varepsilon_{m}^{(k+1)}=D_{m m}$, and $P$ is used to recombine the original wavevectors by setting $R=\Phi P$ in Eq. (10). Because $P^{T} \Phi^{T} U \Phi P=I$ and $P^{T} \Phi^{T} M \Phi P=D$, Eq. (11) is obeyed.

The above approach is formulated for the case of a linear eigenproblem and in the absence of local mesh refinement. The following subsections describe how to apply it to the nonlinear Kohn-Sham equation in the presence of local mesh refinement.

\subsection{Discretization of the Kohn-Sham equation}

In this section, we discuss the discretization of the Kohn-Sham equation on a locally refined set of grids and specifically the scaling of the discretization errors with the mesh spacing. In standard multigrid solutions of linear equations, the discretization errors arise only from the finest grid level, $h=h_{\min }$, and, far enough from the domain boundary, scale as $O\left(h_{\min }^{p}\right)$ where $p$ is the order of the Laplace discretization. When employing local mesh refinement, the finest mesh spacing becomes a function of space, denoted by $h_{\min }(\mathbf{r})$ and illustrated in Figure 1 for the case of the water molecule. This implies that the discretization errors are distributed in our case over all grid levels and scale in general as as $O\left(h_{\min }^{p}(\mathbf{r})\right)$. Below we describe the scaling of additional discretization errors that are more specific to our problem. The sources of these additional errors are the boundaries between two grid levels, referred to as inner boundaries, the outer boundary of the coarsest grid, the discretization of the Coulomb potential and the discrete integration scheme.

Inner boundary errors: In the present work we consider a symmetric Laplace discretization of even order. The Laplace operator of order $p$ is represented by the standard stencil, which involves the central grid point and $p$ additional ones in each of the three axes. ${ }^{33}$ This discretization induces errors that scale as $O\left(h^{p}\right)$, where $h$ is the grid spacing. The evaluation of the Laplace operator on the fine grid points adjacent to the inner boundary requires the definition of $p / 2$ auxiliary inner- 


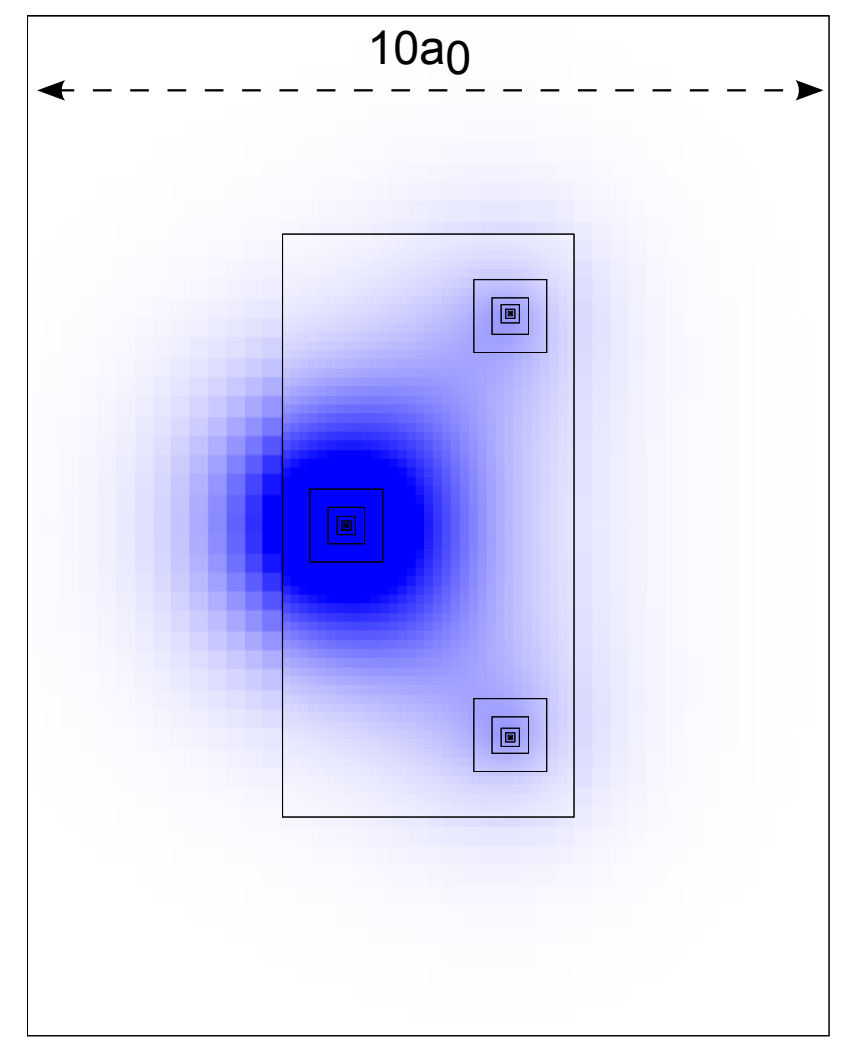

Figure 1: Two-dimensional cross section of the grid hierarchy used for the initial approximation of the $\mathrm{H}_{2} \mathrm{O}$ molecule, presented on top of the electron density distribution, $\rho(\mathbf{r})$. The solid lines represent the inner-boundaries between two refinement patches. The coarsest mesh spacing depicted here is $h=0.125 a_{0}$, where $a_{0}$ is the Bohr radius. The coarsest grid level used in the calculation has a mesh spacing of $h=32 a_{0}$ and extends over a cube, whose edge measures $800 a_{0}$. The mesh spacing of the finest grid level spanned around each atom is $h=2^{-14} a_{0} \approx 6.1 \cdot 10^{-5} a_{0}$. 
boundary grid points (small white points, $a$ and $b$, in Figure 2). Similarly in three dimensions, one has to define $p / 2$ inner-boundary layers around the box-shaped refinement patch. The values of the inner-boundary points are interpolated from the coarser grid level, together with the coarse-tofine correction defined in Eq. (8). This interpolation, illustrated in Figure 2a, introduces additional discretization errors into the Laplace operator. The use of a polynomial interpolation of order $q$ creates errors of order $O\left(h^{q}\right)$, where $h$ is the fine mesh spacing. These errors then enter into the fine equations with a factor of $h^{-2}$ from the Laplace operator. The errors are found in a layer which consists of an $O(h)$ part of entire grid volume, thus causing an overall $O\left(h^{q-1}\right)$ error in global quantities such as the Kohn-Sham eigenvalues. This implies that in order to maintain a uniform $O\left(h^{p}\right)$ scaling of the local discretization errors, the interpolation to the inner-boundary grid points has to be of order $q \geq p+1$. This conclusion was confirmed by numerical tests of the quantum harmonic oscillator problem $\left(V_{i o n}(r)=r^{2}\right.$ and $\left.V_{H}=V_{x c}=0\right)$, which has a known analytical solution and does not involve the effects of a diverging potential. We added a relatively small local refinement grid and tested whether the error in the eigenvalues induced by this grid vanishes with respect to the errors from the bulk as $h$ is reduced systematically. For a Laplace discretization of order $p=4$, this was found to be true only when using an interpolation operator of order $q=6$ or higher (when considering only even-order interpolation operators).

The discretization errors discussed in the previous paragraph can in fact be regarded as the result of the deformation of the original Laplace stencil in fine grid points adjacent to the inner boundary. A similar deformation can be caused by fine-to-coarse transfer operator of the approximation vector, $I_{h}^{2 h}$ in Eq. (5). In general, the fine-to-coarse transfer operator used in the present work is the full-weighting operator, defined as the inverse of the coarse-to-fine linear interpolation. The one-dimensional version of this operator is sketched in Figure 2b, which depicts the transfer of the defect-correction vector, performed using $\hat{I}_{h}^{2 h}$ in Eq. (7). The full-weighting operator yields smoother coarse-grid equations in comparison to the injection operator (direct transfer only between overlapping grid points), which allows for a faster convergence of the GS method. ${ }^{20}$ In our case, this is especially true in the core-regions where the wavefunctions vary significantly in com- 

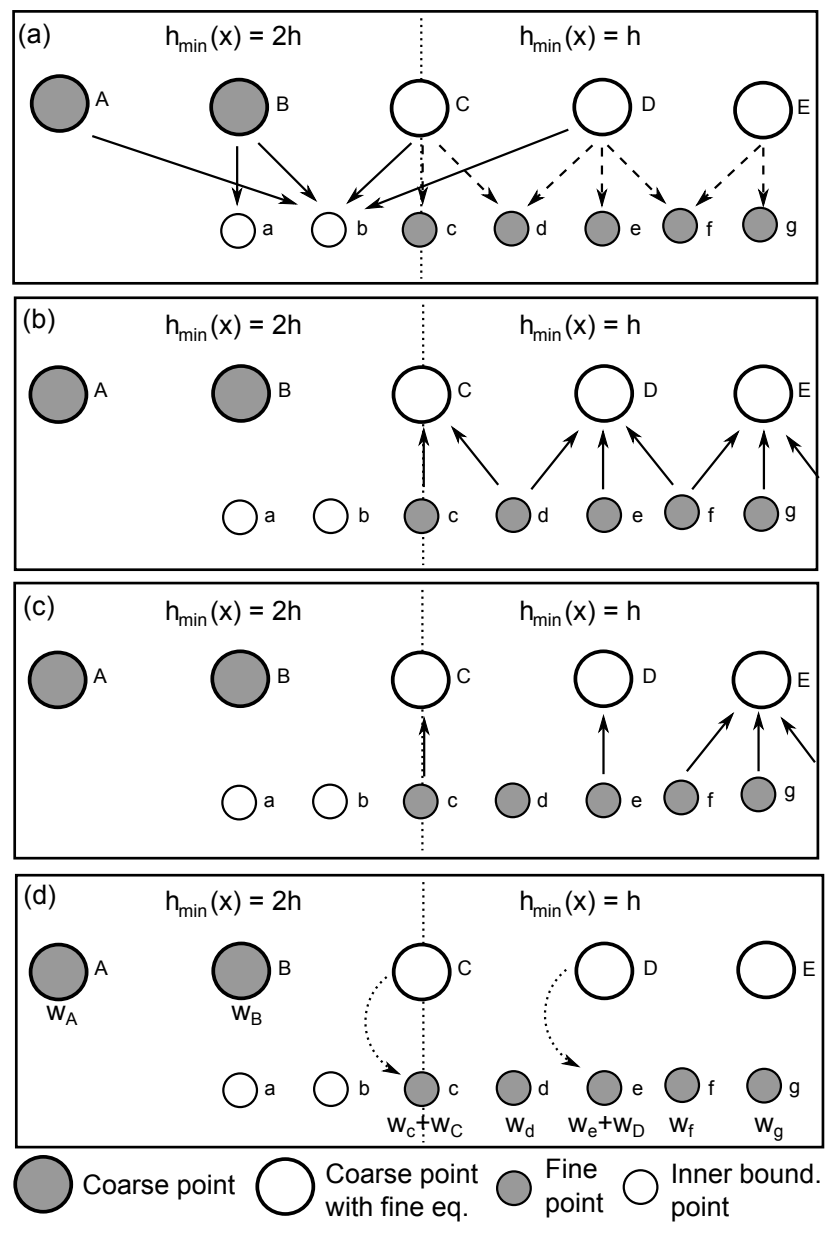

Figure 2: A schematic one-dimensional view of various grid operations in the vicinity of an inner boundary when using a Laplace discretization of order $p=4$ (5 points stencil). (a) Coarse-tofine interpolation: the dashed lines denote the linear order interpolation of the corrections to the approximation vector $\left(I_{2 h}^{h}\right.$ in Eq. (8)), while the solid lines denote the cubic order interpolation of the inner-boundary points; (b) Defect-correction transfer using the full-weighting operator $\left(\hat{I}_{h}^{2 h}\right.$ in Eq. (7)); (c) Transfer of the approximation vector using the full-weighting operator in the bulk and the injection operator in the two layers adjacent to the inner boundary $\left(I_{h}^{2 h}\right.$ in Eq. (5)); (d) Weights used for the discrete integration over the finest available grid level in the entire domain. 
parison to the mesh spacing. However, using the full-weighting operator sketched in Figure $2 b$, for the fine-to-coarse transfer of the approximation vector, defined in Eq. (5), induces discretization errors in the coarse layers adjacent to the inner boundary (points $A$ and $B$ in Figure 2 for a Laplace discretization of order $p=4)$. This is because the stencil describing the Laplace operator at points $\mathrm{A}, \mathrm{B}$ is then different from that of the standard stencil at grid level $2 h$. The resulting discretization errors in the approximation vectors scale as $O\left(h^{2}\right)$. They enter into the coarse equations with an $h^{-2}$ factor from the Laplace operator in layers that comprise an $O(h)$ part of the grid volume. The resulting errors in the eigenvalues therefore scale as $O(h)$, as confirmed by numerical tests. In order to avoid this problem and achieve a uniform $O\left(h^{p}\right)$ scaling of the errors, the wavevectors are transferred by injection from $p$ layers of fine grid points adjacent to the inner boundary. This modification is demonstrated by the injection from point $c$ to $C$ and from point $e$ to $D$ in Figure 2c . Because the wavevectors are smooth in comparison with the mesh spacing close to the edge of the refinement patch, the injection operator can be used there without harming the convergence of the multigrid process.

Integration errors: Another source of discretization errors is the integration scheme employed when evaluating global variables such as the elements of the Ritz matrix, $M_{i j}$, in Eq. (12). In order to obtain the most accurate value, this integration is done using grid points that correspond to finest available mesh spacing, $h_{\min }(\mathbf{r})$. We compute the integration weights associated with each grid point by considering a polynomial interpolation of order $q$ of a general vector, denoted by $\mathbf{T}$, which yields a continuous function, denoted by $T(\mathbf{r})$. In order to integrate the finest mesh spacing, the value at $\mathbf{r}$ is interpolated only from grid level $h_{\min }(\mathbf{r})$. By integrating $T(\mathbf{r})$ over the entire space we obtain a discrete sum over the elements of $\mathbf{T}$, given by

$$
\int d^{3} r T(\mathbf{r})=\sum_{i} w_{i} T_{i}
$$

where the resulting weights, $w_{i}$, define the discrete integration scheme. The errors induced by this scheme are due to the polynomial interpolation and hence scale as $O\left(h^{q}\right)$. Because $w_{i}$ are 
calculated once and for all, upon setting the grid formation, $q$ can be chosen to be higher than that of the Laplace discretization, $p$, with negligible computational cost. We thus chose $q=p+2$. Interpolating $T(\mathbf{r})$ using grid level $h_{\min }(\mathbf{r})$ implies that several coarse grid points, close to the inner boundary in the region spanned by the fine grid level (points C, D in Figure 2d), are also assigned integration weights. In practice, we transfer their weights to the corresponding fine grid points, as depicted by the arrows in Figure 2d. In principle, the same should have applied to the inner-boundary grid points (a, b in Figure $2 d$ ), but this is avoided by obtaining $T(\mathbf{r})$ in the fine grid points adjacent to the inner boundary (points b,c and e) using an asymmetric polynomial interpolation which involves only the fine grid points.

The above integration scheme, which uses the finest available mesh spacing, is denoted here and below as \langle\rangle . In addition to it, we use a different integration scheme in order to compute global variables during the V-cycle on a single grid level. The weights of this coarse integration scheme on grid level $h$, denoted by \langle\rangle$_{h}$, are obtained by adding weights from grid levels finer than $h$ to their corresponding coarse grid points using the full-weighting operator. In Figure $2 \mathrm{~d}$ the definition of $\langle\quad\rangle_{2 h}$ would imply that the weights of points $c, d, e, f$ and $g$ are added to points $C, D$ and $E$ using the full-weighting operator illustrated in Figure $2 \mathrm{~b}$. This integration scheme does not take into account, however, regions of space that are covered only by coarser grid levels. This issue is solved by adding coarse correction to the integral, as discussed in Eq. (2.4).

Potential discretization errors: We conclude this section by discussing the discretizaion of the Coulomb potential. The naive real-space discretization of the singular ionic potential, whereby $V_{i o n, i}^{h}=V_{i o n}\left(\mathbf{r}_{i}\right)$, provides a bad approximation of the continuous Kohn-Sham equation in the vicinity of the nuclei and may lead to a very large condition number. We therefore use a smooth approximation of the Coulomb potential near the cores, obtained by averaging it over a cube around each grid point. This potential is defined as

$$
V_{a}^{h}\left(\mathbf{r}_{i}\right)=\left\{\begin{array}{cc}
\frac{1}{\left|\mathbf{r}_{i}\right|} & \left|\mathbf{r}_{i}\right|>c h \\
h^{-3} \int_{-\frac{h}{2}}^{\frac{h}{2}} d x \int_{-\frac{h}{2}}^{\frac{h}{2}} d y \int_{-\frac{h}{2}}^{\frac{h}{2}} d z \frac{1}{\left|\mathbf{r}_{i}+\mathbf{x}\right|} & \text { else }
\end{array}\right.
$$


where $\mathbf{r}_{i}$ is the distance of the grid point from the position of the atom. The cutoff parameter is chosen in our case to be $c=3$. The cutoff assures that away from the singularity the Coulomb potential is represented by its value at the location of each grid point, similarly to all other quantities in the problem. The above integral can be evaluated analytically using the Gauss theorem, which yields

$$
\begin{aligned}
\int \frac{d^{3} x}{\left|\mathbf{r}_{i}+\mathbf{x}\right|} & =\frac{1}{2} \int \frac{\mathbf{r}_{i}+\mathbf{x}}{\left|\mathbf{r}_{i}+\mathbf{x}\right|} \cdot \hat{n} d^{2} x \\
& =\sum_{\mathbf{x}} \sum_{p} \frac{x}{h / 2} \xi_{x}\left[\xi_{y} \log \left(\xi_{z}+|\xi|\right)-\frac{\xi_{x}}{2} \arctan \left(\frac{\xi_{y} \xi_{z}}{\xi_{x}\left|\xi_{y}\right|}\right)\right]
\end{aligned}
$$

where $\xi=\mathbf{r}_{i}+\mathbf{x}, \sum_{\mathbf{x}}$ runs over the corners of the cube and $\sum_{p}$ denotes all permutations over the $x, y, z$ indices. The total ionic potential is given by a sum of Eq. (14) over all the atoms,

$$
V_{i o n, i}^{h}=\sum_{a} Z_{a} V_{a}^{h}\left(\mathbf{x}_{i}-\mathbf{R}_{a}\right)
$$

where $Z_{a}$ and $\mathbf{R}_{a}$ are the charges and positions of atoms, respectively. Numerical tests indicate that this approximation of the true potential induces local discretization errors in the eigenvalues that scale as $O\left(h^{2}\right)$ near the core. These errors, however, decrease sharply with the distance from the core. We therefore reduce them by greatly refining small regions around the nuclei, as illustrated in Figure 1. Since these additional refinement patches are small (cubic patches of size $17^{3}$ grid points), the computational cost of this solution is relatively small. The precise manner by which the sizes and shapes of the refinement regions are determined is described in Section 2.5.

\subsection{Relaxation Methods}

In the present work, the Kohn-Sham equation, as well as the Poisson equation used for evaluating the Hartree potential, are computed using a more efficient generalization of the lexicographic Gauss-Seidel method (4), known as the red-black Gauss Seidel (RBGS) method (see section 3.4 in Brandt ${ }^{20}$ ). In the RBGS method, the relaxation sweep is performed first over all grid points 
with odd indices and then over all grid points with even indices (in $d$ dimensions the sum of the $d$ indices should be odd or even, respectively). The RBGS relaxation sweeps converge for positivedefinite matrices such as the one corresponding to the Laplace discretization. ${ }^{34}$ The presence of the Coulomb potential yields a small number of 'binding' eigenvectors with negative eigenvalue, whereas most of the eigenvalues of the discrete operator remain positive. This slight non-positivedefiniteness causes smooth error components of the approximation vector, that are proportional to the 'binding' eigenvectors, to diverge slowly when employing the RBGS method. However, on all grid levels except for the coarsest one, this divergence is canceled by the coarse-grid corrections. On the coarsest grid level, where we cannot rely on a yet coarser grid level, we employ instead of the RBGS method the Kaczmarz method, which converges even for non-positive-definite operators, albeit more slowly than RBGS. ${ }^{36}$ The key difference between the two methods is that in each Kaczmarz iteration over a single grid point we alter the value of several neighbouring grid points simultaneously. The properties of the Kaczmarz method and similar distributive methods are further discussed in section 3.4 in Brandt ${ }^{20}$ and references therein. Because the role of the coarsest grid level is in fact to solve the equation to very low residuals, as discussed below Eq. (8), we employ in every V-cycle about 200 Kaczmarz relaxation sweeps on the coarsest grid, as compared to only 4 RBGS sweeps on each of the finer grids.

The Coulomb potential causes a similar problem on finer grid levels in the vicinity of the atoms, where the numerical values of the potential are comparable with the coefficient of the Laplace discretization. As in the case of the coarsest grid level, this issue can be solved using the Kaczmarz method. This is done by performing about 30 Kaczmarz relaxation sweeps on each fine grid in cubic regions of size $7^{3}$ grid points around the location of each atom, instead of the RBGS relaxations. This region is increased during the relaxation to $11^{3}$ grid points in order to prevent the creation of high residuals on a single surface. This local relaxation is in accordance with the idea of performing extra relaxation sweeps in regions that contain high residuals, proposed by Bai and Brandt. ${ }^{37}$

It is worth noting that the amount of computer power invested in the large number of Kaczmarz 
relaxation sweeps during each $V$-cycle is comparable to that invested in the RGBS relaxation sweeps. This is because the overall number of grid points relaxed using the Kaczmarz method is relatively small.

\subsection{One-shot V-cycle of a nonlinear system}

The Kohn-Sham equation is in fact a set of nonlinear equation. We first write down this set of equations, while repeating for the sake of completeness several equations mentioned above, and then discuss a self-consistent approach for solving them. The set of equations include the KohnSham equation of each orbital, defined as

$$
\left(-\frac{\nabla^{2}}{2}+V_{i o n}(\mathbf{r})+V_{H}[\rho ; \mathbf{r}]+V_{x c}[\rho ; \mathbf{r}]\right) \varphi_{k}(\mathbf{r})=\varepsilon_{k} \varphi_{k}(\mathbf{r})
$$

with a Dirichlet boundary condition, $\varphi_{k}=0$, on the outer boundary of the domain. These equations are nonlinear due to the dependence of the potentials on electron density. The definition of the latter, presented also in Eq. (2), forms an additional equation in the set, given by

$$
\rho(\mathbf{r})=\sum_{k} f_{k}\left|\varphi_{k}(\mathbf{r})\right|^{2}
$$

where $f_{k}$ is the occupation number of wavevector $k$. The occupation numbers are set according to the aufbau principle, i.e., from the lowest energy and up. Another equation is due to the conservation of charge, which implies that the wavevectors have to be normalized and hence

$$
\int d \mathbf{r}\left|\varphi_{k}(\mathbf{r})\right|^{2}=1
$$

The exchange-correlation potential in Eq. (17) is a functional of $\rho$ and is defined as the functional derivative of the exchange-correlation functional,

$$
V_{x c}[\rho ; \mathbf{r}]=\frac{\delta}{\delta \rho} E_{x c}[\rho]
$$


In the present work, we implement the simplest approximation for $E_{x c}$, known as the local density approximation (LDA), in which $V_{x c}$ is taken to be a local function of $\rho(\mathbf{r}) .{ }^{38,39}$ The Hartree potential is also a functional of $\rho$, obtained by solving the Poisson equation,

$$
\nabla^{2} V_{H}=-4 \pi \rho(\mathbf{r})
$$

using the RBGS method. This Poisson equation has a nontrivial Dirichlet boundary condition on the edge of the domain, which is approximated by a multipole expansion, ${ }^{40,41}$

$$
V_{H}(r, \theta, \phi)=\sum_{l=0}^{l_{\max }} \sum_{m=-l}^{l} \frac{4 \pi}{2 l+1} \frac{1}{r^{l+1}} Y_{l, m}(\theta, \phi) Q_{l m}
$$

Here $Y_{l m}$ are the spherical harmonic functions and the $Q_{l m}$ coefficients are given by

$$
Q_{l m}=\int d r d \theta d \phi r^{l} Y_{l, m}(\theta, \phi) \rho(r, \theta, \phi)
$$

In the present work we chose $l_{\max }=9$, which has been shown to provide sufficient precision by comparing the results obtained in the present work to those obtained using a prolate-spheroidal grid. ${ }^{16,17}$

Equations (17)-(23) form a set of nonlinear equations which can be solved using a self-consistency cycle. A naive cycle would consist of solving Eq. (17) to very low residuals (through several $V$ cycles), followed by an update of $\rho, V_{x c}$ and the solution of Eq. (21), with $\rho$ fixed, to very low residuals. A great advantage of iterative solvers is that they allow one to perform a more efficient self-consistency cycle whereby each equation is not solved to very low residuals in each self-consistency step. Such an efficient cycle has been implemented within the DFT framework by Kohn et al., ${ }^{25}$ who recalculated the potentials after only a single V-cycle of Eq. (17). One can take this idea one step further and update the potentials on each grid level after every relaxation sweep on that level, following an approach known as the one-shot V-cycle. ${ }^{42}$ By creating high coupling between the components of the nonlinear equation, the one-shot V-cycle is able to converge much 
faster than the alternating cycle, where the potentials are updated only once per V-cycle after the Rayleigh-Ritz process. We confirmed this by numerical tests for the hydrogen atom, where the one-shot V-cycle was found to converge 5 to 10 times faster than the alternating cycle.

For linear equations and in the alternating cycle, the potentials in Eq. (17) are simply transferred to the coarse grid levels from the finest one by either the injection or the full-weighting operator. In the one-shot V-cycle, however, the whole set of equations, (17)-(23), has to be transferred to the coarse grid levels according to the FAS scheme, described in Section 2.1.

The FAS scheme takes a slightly different form for an integral equation such as the normalization condition, defined in Eq. (19). The latter has to be maintained during the one-shot V-cycle because of the recalculation of the electron density according to Eq. (18), which is true only for normalized Kohn-Sham orbitals. Because on all grid levels, excluding the finest, the Kohn-Sham equation is in fact nonhomogeneous due to the fine-to-coarse defect correction, the normalization of the wavevectors has to be accompanied by an update of the eigenvalues. The recalculation of the eigenvalues can be done using the Rayleigh quotient formula,

$$
\varepsilon_{k}=\frac{\int d \mathbf{r} \varphi_{k}^{\star}(\mathbf{r})\left(-\frac{\nabla^{2}}{2}+V_{i o n}(\mathbf{r})+V_{H}[\rho ; \mathbf{r}]+V_{x c}[\rho ; \mathbf{r}]\right) \varphi_{k}(\mathbf{r})}{\int d \mathbf{r}\left|\varphi_{k}(\mathbf{r})\right|^{2}}
$$

which is obtained by integrating over Eq. (17).

Following the FAS scheme, we transfer the fine-grid corrections to the integrals in the above equation and in Eq. (19). For a general integral over the entire domain, $\int d \mathbf{r} F(\mathbf{r})$, the fine-grid corrections to level $h$ are given by

$$
\tau_{F}^{h / 2, h} \equiv\left\langle F^{h / 2}\right\rangle_{h / 2}-\left\langle I_{h / 2}^{h} F^{h / 2}\right\rangle_{h}+\tau_{F}^{h / 4, h / 2}
$$

where the inner-product is evaluated using the coarse integration scheme defined in Section 2.2, and $\tau_{F}^{h / 4, h / 2}$ denotes a similar correction transferred from grid level $h / 4$ to grid level $h / 2$. In addition, we need to transfer a coarse-grid correction to $\int d \mathbf{r} F(\mathbf{r})$, that accounts for the contribution to the integrals from regions of space that are covered only by grid levels that are coarser than $h$. This 
correction is defined as

$$
\tau_{F}^{2 h, h} \equiv \sum_{i \in 2 h} F_{i}^{2 h} w_{i}+\tau_{F}^{4 h, 2 h}
$$

where $w_{i}$ denotes the fine-integration weights. The weights for grid points on grid level $2 h$ that have corresponding grid points on grid level $h$ (see Figure $2 \mathrm{~d}$ ). The correction from grid level $2 h$ also includes coarse-grid corrections from a yet coarser grid level, $\tau_{F}^{4 h, 2 h}$, which vanishes when $2 h$ is the coarsest grid level. It is useful to note that the coarse-grid corrections vanish in standard multigrid implementations (without local mesh refinement). Using these corrections the integral is approximated on grid level $h$ as,

$$
\int d \mathbf{r} F(\mathbf{r}) \approx\left\langle F^{h}\right\rangle_{h}+\tau_{F}^{h / 2, h}+\tau_{F}^{2 h, h}
$$

Following the above formulation, we can define the normalization factor, $\alpha_{k}^{h}$, of the $k^{t h}$ KohnSham orbital on grid level $h$ as

$$
\left(\alpha_{k}^{h}\right)^{2}\left(\left\langle\varphi_{k}^{h}, \varphi_{k}^{h}\right\rangle_{h}+\tau_{\varphi_{k} \varphi_{k}}^{2 h, h}\right)+\tau_{\varphi_{k} \varphi_{k}}^{h / 2, h}=1
$$

where $\tau_{\varphi_{k} \varphi_{k}}^{h / 2, h}$ and $\tau_{\varphi_{k} \varphi_{k}}^{2 h, h}$ are the fine-grid and coarse-grid corrections to the integral in Eq. (19), respectively. This normalization factor is applied by setting

$$
\varphi_{k}^{h^{\prime}} \rightarrow \alpha_{k} \varphi_{k}^{h^{\prime}} \quad \tau_{\varphi_{k} \varphi_{k}}^{h^{\prime}, h^{\prime} / 2} \rightarrow \alpha_{k}^{2} \tau_{\varphi_{k} \varphi_{k}}^{h^{\prime}, h^{\prime} / 2}
$$

on grid level $h$ and all the coarser grid levels, $h^{\prime} \geq h$. In order to save computational work the actual multiplication of wavevectors on coarser levels is performed only upon entering each grid level. Similarly, the eigenvalues are updated according to Eq. (24) as

$$
\varepsilon_{k}=\frac{\left\langle\varphi_{k}^{h}, H^{h} \varphi_{k}^{h}-\tau_{k}^{h}\right\rangle_{h}+\tau_{\varphi_{k} H \varphi_{k}}^{2 h, h}+\tau_{\varphi_{k}\left(H \varphi_{k}-\tau_{k}\right)}^{\frac{1}{2} h, h}}{\left\langle\varphi_{k}^{h}, \varphi_{k}^{h}\right\rangle_{h}+\tau_{\varphi_{k} \varphi_{k}}^{2 h, h}+\tau_{\varphi_{k} \varphi_{k}}^{\frac{1}{2} h, h}}
$$

where $\tau_{k}^{h}$ is the defect-correction vector of the Kohn-Sham equation, which is not to be confused 
with the fine-grid and coarse-grid corrections to the numerator of Eq. (24), denoted by $\left.\tau_{\varphi_{k}(H / 2, h}^{h /} \varphi_{k}-\tau_{k}\right)$ and $\tau_{\varphi_{k} H \varphi_{k}}^{2 h, h}$, respectively. For a wavevector that has been normalized by the factor defined in Eq. (28), the denominator in Eq. (30) is in fact equal to 1.

It is important to note that in usual applications of local mesh refinement we would need to enforce global equations, such as the normalization of the wavevectors, only on very coarse grids that span a significant portion of the domain. In our case, however, due to the singular potential, some wavevectors are highly localized around the atoms. This implies that even grids that span a relatively small volume around a single atom may still represent a significant portion of a wavevector and thus require the relaxation to be accompanied by a normalization of the relevant wavevectors and an update their eigenvalues.

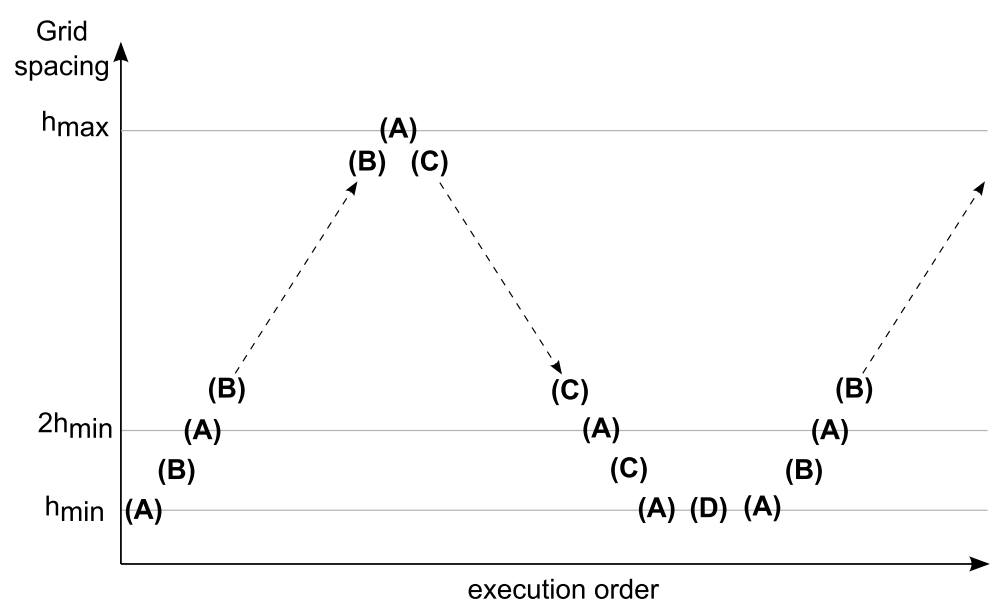

Figure 3: Schematic view of the one-shot V-cycle. Details of each step are provided in the text.

The procedures employed during the one-shot V-cycle are summarized in Figure 3. The steps in the diagram are defined as follows:

(A) Relaxation: Relax $\left\{\varphi_{k}^{h}\right\}$, normalize $\left\{\varphi_{k}^{h}\right\}$, calculate $\rho$, calculate $V_{x c}$, relax $V_{H}$ and update $\left\{\varepsilon_{k}\right\}$.

(B) Fine-to-coarse transfer: Transfer $\left\{\varphi_{k}^{h}\right\}$, transfer $V_{H}$, transfer defect corrections to $\rho, V_{x c}$, $V_{H},\left\{\varphi_{k}^{h}\right\}$ and global variables $\left(Q_{l m}, \alpha_{k}\right.$, and $\left.\varepsilon_{k}\right)$.

(C) Coarse-to-fine correction: Correct $\left\{\varphi_{k}^{h}\right\}$ and $V_{H}$, transfer corrections to $\alpha_{k}$ and $\varepsilon_{k}$. 
(D) Rayleigh-Ritz process: Perform a modified Rayleigh-Ritz process (see Section 2.7) followed by a recalculation of the coarse-to-fine corrections to $\alpha_{k}$ and $\varepsilon_{k}$.

The one-shot V-cycle performs well for small systems such the hydrogen atom. However, in many electron systems it was found to exhibit a very slow convergence of the residuals or even illconvergence in some of the cases. A careful study of the residuals suggested that the order of operations on a single grid level, which alternates between relaxation sweeps of the Kohn-Sham equation and updating of the potentials, did not provide enough coupling between the components of the nonlinear set of equations. One approach to increase the coupling between components of an ill-converging self-consistency cycle is through mixing of components from previous steps. In this work we employ the generalized Broyden method ${ }^{43}$ which has been successfully implemented in the past in electronic structure calculations. ${ }^{44,45}$ We used this method in order to yield a new approximation of all the occupied wavevectors (for which $f_{k} \neq 0$ ), $V_{x c}$, and $V_{H}$, by mixing each vector with its values from several previous relaxation sweeps. The weights of mixing are chosen such that they minimize the overall residual of the Eqs. (17),(18) and (21). The mixing is used only in combination with the Kaczmarz relaxation on the coarsest grid level and on finer ones in the vicinity of the atoms, and hence its additional computational cost is relatively small. Mixing was found to accelerate and sometimes enable the convergence of the many-electron systems discussed in Section 3.

The effect of the mixing is presumably to reduce smooth components that diverge as a result of the combination of relaxation sweeps and potential updates. The mixing algorithm is able to single out these components from the difference between the current approximation vector and its values in previous iterations, and reduce them from the current vector in a manner that minimizes the overall residual of the set of nonlinear equation.

\subsection{Grid refinement via a $\lambda$-FMG cycle}

As with any self-consistent cycle, the one-shot V-cycle, described in Section 2.4, has to start from an initial guess which is inside its basin of convergence. The multigrid approach provides an 
inherent method for obtaining such an approximation, known as the Full Multigrid (FMG) algorithm. ${ }^{20,21}$ We first describe the original algorithm, formulated within the standard multigrid framework, where $h_{\min }$ is homogenous in space. The FMG cycle begins from a single coarse grid or a small set of coarse grids where the initial approximation can be inexpensively obtained using relatively costly methods such as a direct diagonalization of the discrete equations. Each FMG iteration consists of several $V$-cycles followed by an interpolation of the current approximation into a new grid level whose mesh spacing is half of the currently finest mesh spacing, $h_{\min } \rightarrow h_{\min } / 2$. These iterations are repeated until the discretization errors, whose estimation is discussed below, have been reduced below a desired level. The generalization of this concept to the case of locallyrefined multigrids is known as the $\lambda$-FMG cycle (see section 9.6 in Brandt $^{20}$ ). According to this approach, every several V-cycles $h_{\min }(\mathbf{r})$ is refined in various regions of space, which can be automatically chosen in a manner that yields a desired increase in accuracy using the minimal amount of computational work.

Unlike in the textbook examples of the $\lambda$-FMG cycle, here even the initial set of coarse grids has to include many small refinement grids around each atom, in order to provide an accurate enough discretization of the singular potential and oscillatory wavevectors. This initial set is illustrated for the case of the $\mathrm{C}_{6} \mathrm{H}_{6}$ molecule in Figure $4 \mathrm{a}$, which shows 10 grids that span the entire molecule and 10 additional small refinement grids around each atom. Because of their small size $\left(17^{3}\right.$ points each), the fraction of the total computational work invested in these refinement grid is relatively small.

As mentioned above, the refinement of $h_{\min }(\mathbf{r})$ during the $\lambda$-FMG cycle should yield a desired accuracy with the minimal amount of work. The total work, denoted by $W\left[h_{\min }(\mathbf{r})\right]$, is assumed to be proportional to the total number of grid points,

$$
\mathscr{W}\left[h_{\min }(\mathbf{r})\right] \propto \int d^{3} r h_{\min }^{-3}(\mathbf{r})=\sum_{i} w_{i} h_{\min }^{-3}\left(\mathbf{r}_{i}\right)
$$

where $i$ runs over all the grid points and $w_{i}$ are the fine-integration weights, discussed in Section 

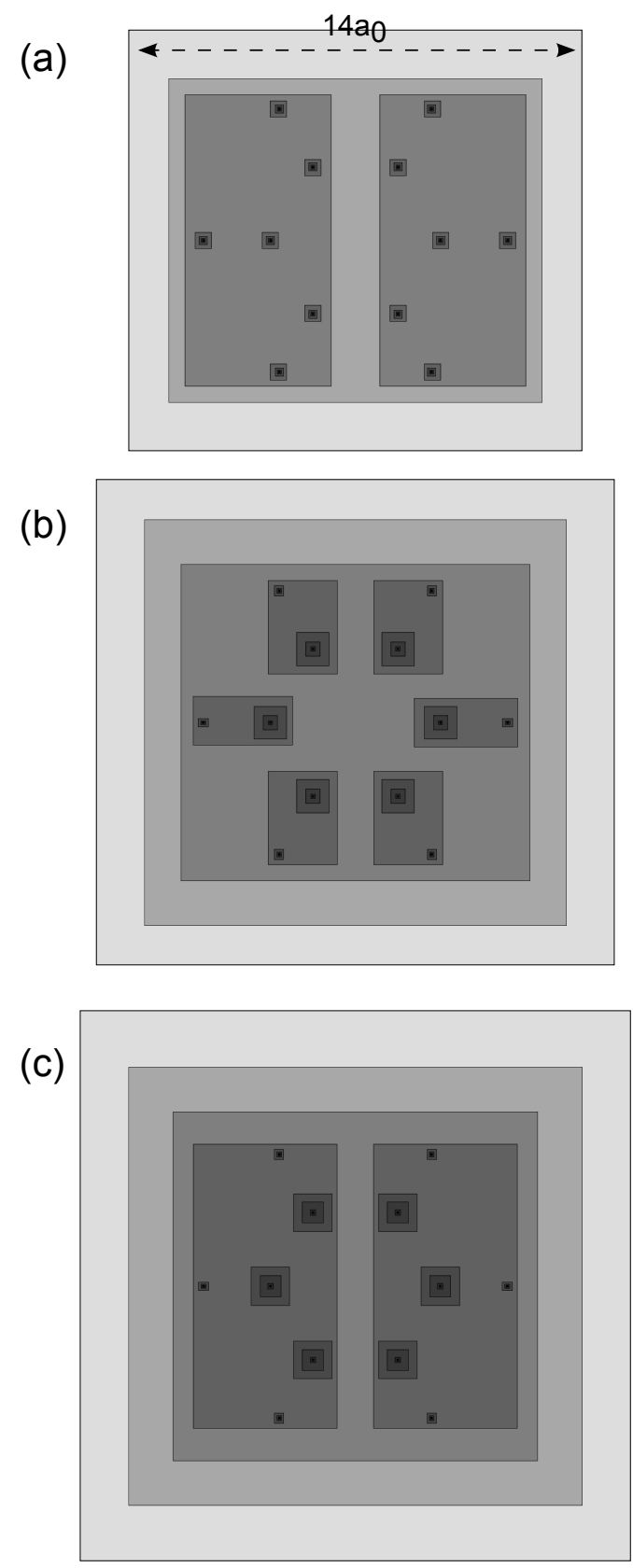

Figure 4: Two-dimensional cross sections of the grid hierarchy during various $\lambda$-FMG steps in the calculation of the $\mathrm{C}_{6} \mathrm{H}_{6}$ molecule. The gray-scale represents the mesh spacing, whereby darker areas corresponds to finer grids. The coarsest grid presented here has a mesh spacing of $h=0.25 a_{0}$. Several coarser grid levels are not represented, out of which the coarsest is a cube whose edge measures $800 a_{0}$ and its mesh spacing is $h=32 a_{0}$. The grid formation during the $1^{s t}, 4^{t h}$ and $6^{\text {th }}$ $\lambda$-FMG steps are shown in figure (a), (b), and (c), respectively. 
2.2. In the present work we chose to derive our estimate for the discretization errors from the Kohn-Sham total energy, defined as

$$
E_{K S}=E_{x c}[\rho]+\int d^{3} r \sum_{k} f_{k} \varphi_{k}(\mathbf{r})\left(-\frac{\nabla^{2}}{2}+V_{i o n}(\mathbf{r})+V_{H}[\rho ; \mathbf{r}]\right) \varphi_{k}(\mathbf{r})
$$

The total discretization error is assumed to be an integral over a local error estimator, $\mathscr{E}\left[h_{\min }(\mathbf{r})\right]=$ $\int d^{3} r g\left(h_{\min }(\mathbf{r}), \mathbf{r}\right)$, where, as suggested by Brandt, ${ }^{20} g\left(h_{\min }(\mathbf{r}), \mathbf{r}\right)$ is estimated for grid level $h$ from the defect correction to the Kohn-Sham energy in grid level $2 h$. The estimator can thus be written as

$$
\mathscr{E}\left[h_{\min }(\mathbf{r})\right]=\sum_{i} w_{i} g\left(h_{\min }\left(\mathbf{r}_{i}\right), \mathbf{r}_{i}\right) \approx \sum_{i} w_{i} G\left(\mathbf{r}_{i}\right)\left(2^{p\left(\mathbf{r}_{i}\right)}-1\right)^{-1}\left|\left(\mathbf{e}_{K S}^{h}-I_{2 h}^{h} \mathbf{e}_{K S}^{2 h}\right)_{i}\right|
$$

where $\mathbf{e}_{K S}$ is the vector containing the contribution of each grid point to the Kohn-Sham energy, given by the integrand in Eq. (32), $p(\mathbf{r})$ denotes the estimated scaling of the local discretization errors, and $I_{2 h}^{h}$ is chosen to be the linear interpolation operator. The function $G(\mathbf{r})$ is an error weighting function which is approximated to be an unknown constant in space, $G(\mathbf{r}) \approx G_{0}$. The function $p(\mathbf{r})$ is assumed to be equal to the order of the Laplace operator for all the grid points. This would be a wrong estimate only in the finest grid level close to the atoms, where the errors related to the Coulomb potential, which scale as $O\left(h^{2}\right)$, dominate over the errors due to the Laplace discretization. However, this issue does not affect our estimation of the total error since we span a set of very fine grids around each atom, thus assuring that any errors resulting from the finest grid levels are negligible with respect to the overall discretization errors.

As mentioned above, the aim of the $\lambda$-FMG cycle is to find $h_{\min }(\mathbf{r})$ which yields a desired accuracy using the minimal amount of work. One natural approach to this constrained minimization problem is to introduce a lagrange multiplier, $\lambda$, and solve the following equation:

$$
\frac{d \mathscr{E}}{d h_{\min }(\mathbf{r})}=-\lambda \frac{d \mathscr{W}}{d h_{\min }(\mathbf{r})}
$$

When $\lambda$ is high, the amount of work in the solution of Eq. (34) is relatively low. During the $\lambda$ - 
FMG cycle, $\lambda$ is gradually decreased, yielding higher $W$ and hence a finer discretization which leads to a better accuracy.

Each $\lambda$-FMG step consists of several $V$-cycles (chosen here to be 2 ) that are followed by the calculation of $h_{\min }(\mathbf{r})$ from Eq. (34) and interpolation of the wavevectors and Hartree potential to the newly refined regions. The functional minimization in Eq. (34) is done by iteratively refining the existing box-shaped grids. In every iteration we compute for each of the rectangular grids the reduction in the error defined in Eq. (33) and the increase of work due to its expansion by two grid point layers in each of the 6 possible directions. We then select the refinement step which has the highest accuracy-to-work tradeoff, given by $\lambda=-d E / d W$. This process is repeated, ideally until $E\left[h_{\min }(\mathbf{r})\right]$ has been reduced by a desired factor, denoted by $\sigma$, with the interpolation to the newly refined regions performed thereafter. In practice, it is more convenient to stop the refinement when the total number of grid points has increased by a corresponding factor. As demonstrated by Bai and Brandt, ${ }^{37}$ the $\lambda$-FMG cycle should yield an accuracy-to-work ratio similar to that obtained without local mesh refinement using the standard FMG algorithm. In the latter case, since $h_{\min }(r)=h_{\min }$ and hence $E \sim h_{\min }^{p}$ and $W \sim h_{\min }^{-d}$ we obtain that

$$
E \sim W^{-p / d}
$$

where $d$ is the dimension of the system. Assuming that this ratio applies to our problem, we can continue refining the grids until the total number of grid points has increased by a factor of $\sigma^{-d / p}$. As discussed in Section 3 below, the constant decrease in the discretization error by a factor $\sigma$ allows one to estimate the total discretization error. The $\lambda$-FMG cycle can be stopped when the estimated error has gone below the desired level.

For the standard FMG algorithm, where $E \propto h_{\min }^{p}$, the value of the refinement factor is given by $\sigma=2^{-p}$. In the present work, where $p=4$, we chose a more moderate factor of $\sigma=1 / 3$ in order to avoid a refinement of two or more grid levels at certain regions of space during a single $\lambda$-FMG step, which may yield high interpolation errors. The grid hierarchy resulting from the 
above algorithm with $\sigma=1 / 3$ is shown in Figure 4 for the case of the $\mathrm{C}_{6} \mathrm{H}_{6}$ molecule.

\subsection{Initial approximation on coarse grids}

As mentioned above, the $\lambda$-FMG process is performed after obtaining an initial guess of the wavevectors on the initial set of grids. In the present study this solution is obtained through a continuation process, where we relax the Kohn-Sham equation (1) with a modified potential given by

$$
V_{K S}[\rho ; \mathbf{r}]=V_{i o n}(\mathbf{r})+\zeta\left(V_{H}[\rho ; \mathbf{r}]+V_{x c}[\rho ; \mathbf{r}]\right)
$$

where $\zeta$ controls the nonlinearity of the potential $(0 \leq \zeta \leq 1)$. Starting from the linear equation, $\zeta=0$, the parameter $\zeta$ is gradually increased during the continuation process up to $\zeta=1$. The initial approximation of the wavevectors is obtained by spanning around each of the atoms the known hydrogen-like wavefunctions, given by

$$
\psi_{n l m}(r, \theta, \phi)=C e^{-\frac{2 Z r}{n}} L_{n-l-1}^{2 l+1}\left(\frac{2 Z r}{n}\right) Y_{l m}(\theta, \phi)
$$

where $C$ is the normalization factor, $Z$ is the charge of the atom, $L_{q}^{p}(r)$ are the generalized Laguerre functions and $Y_{l m}(\theta, \phi)$ are the spherical harmonic functions. Because these wavevectors are highly localized around their original atom, the existence of other atoms in the system amounts to a small correction to their decay at large distances. They thus provide a good initial approximation for the molecular wavevectors in the case where $\zeta=0$.

Starting from this initial approximation, we iteratively perform a single one-shot V-cycle and increase $\zeta$ until it reaches the value of $\zeta=1$. Since the last stages of the continuation process were found to be more difficult for the self-consistent cycle, we chose to increase $\zeta$ quadratically as

$$
\zeta=2(k / K)-(k / K)^{2}
$$

where $k$ denotes the index of the continuation step $(0 \leq k \leq K)$ and the total number of continuation 
steps was chosen to be $K=10$. This increases gradually the strength of the repulsive interactions between the electrons, causing the atomic wavevectors to delocalize and recombine into the final molecular wavevectors. In complex molecules such as $\mathrm{CO}_{2}, \mathrm{C}_{6} \mathrm{H}_{6}$ and $\mathrm{CH}_{3} \mathrm{COOH}$ we found that it was necessary to perform $3 \mathrm{~V}$-cycles between each increase of $\zeta$ due to the slow convergence of the residuals, discussed in Section 5 below. In addition, it was necessary in these molecules to perform several $V$-cycles for the Hartree potential immediately after the increase of $\zeta$, in order to stabilize the self-consistency cycle against this abrupt change.

\subsection{Modified Rayleigh-Ritz process}

In this section, we discuss a modified Rayleigh-Ritz procedure, which we introduce in order to stabilize the convergence of the one-shot V-cycle and $\lambda$-FMG cycle. In the present work, we use the standard symmetric Laplace discretization. Close to the inner-boundaries, the Laplace stencil involves the auxiliary inner-boundary grid points, whose values are interpolated from the coarse grid points, as illustrated in Figure 2a. When integrating the resulting 'deformed' Laplace discretization, we find that $\left\langle\varphi_{k}, \nabla^{2} \varphi_{l}\right\rangle \neq\left\langle\varphi_{l}, \nabla^{2} \varphi_{k}\right\rangle$ for $k \neq l$, and hence the Ritz matrix, defined in Eq. (12), is asymmetric. Additional asymmetry of the Ritz matrix arises from the use of non-uniform integration weights close to the inner boundary, as illustrated in Figure 2d. Obtaining a recombination of $\left\{\varphi_{k}\right\}$ from the diagonalization of this asymmetric Ritz matrix yields a non-orthogonal recombination of wavevectors with very similar eigenvalues. Due to molecular symmetries, many of the systems we tested included such degenerate orbitals. The resulting nonorthogonality is inconsistent with the definition of $\rho(\mathbf{r})$ in Eq. (2), leading to global discretization errors in the electron density. Nevertheless, these errors are of the same order as the local errors in the wavevectors near the inner boundary, and therefore do not change the scaling of the errors with $h$, discussed in Section 2.2.

A more problematic effect of the asymmetry of the Laplace discretization is that it hampers the convergence of the one-shot $V$-cycle. When the residuals of the Kohn-Sham equations are close to the level of discretization errors, the Rayleigh-Ritz procedure becomes sensitive to changes in $V_{H}$ 
and $V_{x c}$, yielding a significantly different recombination of the degenerate wavevectors after every $V$-cycle, which in turn leads to a significant change in $V_{H}$ and $V_{x c}$. As a result, the residuals of the Kohn-Sham equation could not be reduced below the level of discretization errors, as shown by the solid line in Figure $5 \mathrm{a}$ for the $\mathrm{Li}_{2}$ molecule. Even though convergence to the level of discretization errors is, in principle, all that one needs for obtaining physical results, convergence to very low residuals is still necessary for verifying that various components of the solver function properly. The instability of the one-shot $V$-cycle, shown in Figure 5a, also affects the $\lambda$-FMG cycle. Interpolating the wavevectors and potentials into a new grid level before they are fully converged, causes high algebraic errors, which require in turn many $V$-cycles in order to be removed.

One may obtain orthogonal wavevectors using a naive symmetrization of the Ritz matrix, $\frac{1}{2}\left(M^{T}+M\right)$. However, this approach does not solve our problem, as demonstrated by the dashdotted line in Figure 5a. In addition, this symmetrization yields relatively high residuals (with respect to the discretization errors) for eigenvectors with a relatively high eigenvalue. The type of asymmetry of the Laplace discretization discussed here is seldom mentioned in the literature ${ }^{17,46,47}$ since it is negligible in the absence of many local mesh refinement grids and does not affect convergence of linear eigenproblems (see for instance the dashed line in Figure 5a). Ewing et al. approached this issue by creating a symmetric Laplace discretization near the inner boundaries. ${ }^{48}$ Such a symmetrization scheme is difficult to derive for high order differential operators. In addition, it does not eliminate the asymmetry caused by the integration weights. We therefore take a more generic approach to this problem by defining a modified Rayleigh-Ritz process which enforces orthogonality between degenerate wavevectors. Below we provide a brief account of this procedure, with details given in Appendix A.

The modified process, which we name the constrained asymmetric Rayleigh-Ritz(CARR) procedure, runs iteratively over all the wavevectors. For wavevector $k$, it produces a recombination of the original wavevectors, which minimizes the residuals of the Kohn-Sham equation while maintaining orthogonality with respect to wavevectors with an eigenvalue, $\varphi_{j}$, such that $\left|\varepsilon_{j}-\varepsilon_{k}\right|<\Delta_{\varepsilon}$. Here $\Delta_{\varepsilon}$ is a parameter that should be proportional to the current level of discretization errors. The 

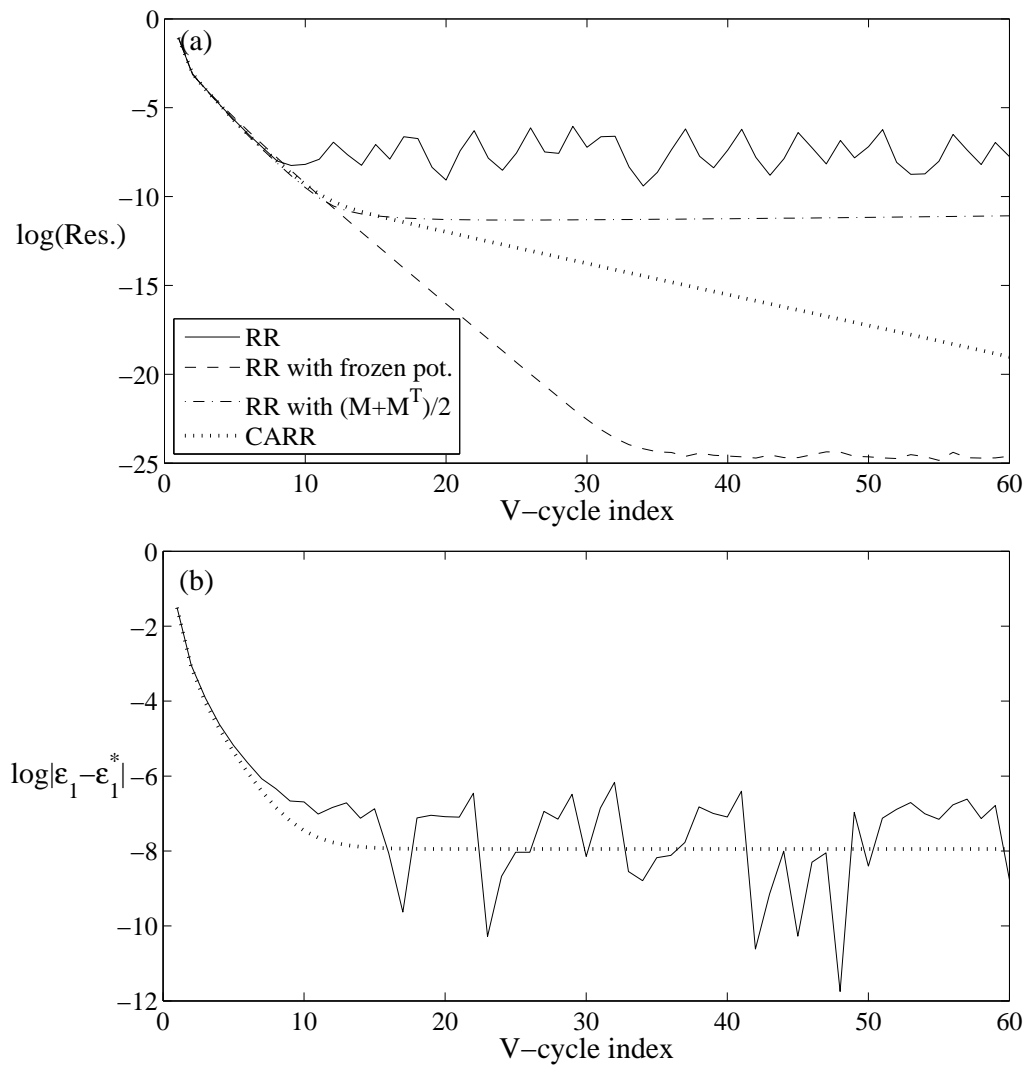

Figure 5: Convergence of the one-shot $V$-cycle with various Rayleigh-Ritz procedures, demonstrated for the $\mathrm{Li}_{2}$ molecule. (a) Logarithm of the residual of the eigenvector with the lowest eigenvalue. (b) $\log \left|\varepsilon_{1}-\varepsilon_{1}^{\star}\right|$, where $\varepsilon_{1}$ is the lowest eigenvalue and $\varepsilon_{1}^{\star}$ is our best estimate of the exact eigenvalue, obtained on a much finer grid formation. The solid and dotted lines correspond to the standard Rayleigh-Ritz and the CARR procedures, respectively. The dash-dotted line corresponds to the the Rayleigh-Ritz procedure performed with the symmetric part of the ritz matrix, $\frac{1}{2}\left(M^{T}+M\right)$. The dashed line corresponds to the standard Rayleigh-Ritz procedure, such that $V_{H}$ and $V_{x c}$ are kept frozen after the first $V$-cycle. 
result is a transformation that minimizes the residuals while maintaining a high level of orthogonality between degenerate wavevectors (nonorthogonality is kept much lower than the level of discretization errors). As a result, the recombination of the degenerate wavevectors is not sensitive to changes in $V_{H}$ and $V_{x c}$, leading to a stable convergence of the residuals, as demonstrated by the dotted line in Figure 5a. Figure Figure 5b shows the difference between the eigenvalue of the lowest eigenvector and its more precise value obtained on a finer grid settings. The figure demonstrates that the CARR procedure does not change the precision in comparison to the usual Rayleigh-Ritz procedure.

We emphasize strongly that one can consider various generalizations of the CARR process that may make it more efficient than the standard Rayleigh-Ritz process and that may prove essential to overall scaling of our approach. This is discussed further in Appendix A

\section{Numerical Results}

In this section, we present numerical results obtained using the above approach, implemented in a program we named CARMA (Concurrent All-electron Real-space Multigrid Algorithm). We first discuss the method used for estimating the discretization errors and analyze its performance in detail for the case of the $\mathrm{H}_{2}$ molecule. We then present high accuracy results for several molecular systems and compare them to corresponding values found in the literature and to results obtained from calculations based on gaussian basis sets.

Before presenting the results obtained for various molecules, we provide some additional information about the practical implementation of the approach discussed in the previous sections.

Exchange-correlation functional : All calculations were performed for the spin-unpolarized Kohn-Sham equation using the local density approximation (LDA) exchange-correlation energy functional, in the parameterized form given by Perdew and Wang. 39 
Transfer operators : The Kohn-Sham and Hartree equations were represented using a $4^{\text {th }}$ order Laplace discretization. As explained in Section 2.2, this requires a discrete integration operator which is based on a $6^{\text {th }}$ order polynomial interpolation and a $6^{\text {th }}$ order interpolation of the innerboundary grid points (sketched in Figure 2a). The same order of interpolation is required when refining the $\left\{\varphi_{k}\right\}$ and $V_{H}$ during the $\lambda$-FMG cycle. In general, fine-to-coarse transfers (operators $I_{h}^{2 h}$ and $\hat{I}_{h}^{2 h}$ in Eqs. (5), (7), and (8)) were performed using the full-weighting operator sketched in Figure 2b. As discussed in Section 2.2, $\left\{\varphi_{k}\right\}$ and $V_{H}$ need to be transferred via injection to grid points close to the inner boundary in order to avoid inducing additional discretization errors (see Figure 2c). Coarse-to-fine corrections (operator $I_{2 h}^{h}$ in Eq. (8)) were transferred using the linear interpolation operator.

Grid structure: In all computations, the coarsest grid had a mesh spacing of $h=32 a_{0}$ and spanned a cubic region around the molecule whose edge measured $800 a_{0}$. By comparison to previous calculations of diatomic molecules, this choice can be shown to yield sufficiently negligible outer-boundary discretization errors. The approach of local mesh refinement allows this distance to be further increased with negligible computational cost, by expanding the coarsest grid or adding a coarser grid level. The initial grid formation included also 5-8 finer grids that spanned the entire molecule and a set of small refinement grids around each atom, such that the finest mesh spacing was $h=2^{-15} a_{0} \simeq 1.6 \cdot 10^{-15} \mathrm{~m}$. The geometries of the molecules are given in Table Table 1 .

$\lambda$-FMG cycle: The results presented below were obtained using the $\lambda$-FMG algorithm described in Section 2.5. One important parameter in this algorithm is $\sigma$, which is taken to be $\sigma=1 / 3$ in order to prevent the possibility of double grid refinement in a single $\lambda$-FMG step (this option is also excluded in the implementation of the algorithm). The number of V-cycles performed in every $\lambda$-FMG step was chosen to be 2 . In choosing this number we had to consider the fact that during each one-shot V-cycle the residuals were reduced by a factor that varied between 2 to 5 . This convergence rate is slow with respect to standard multigrid implementations, as further discussed in Section 5. This suggests, that in order order to maintain the residual below the level of the 
discretization errors (which decrease with the rate $\sigma=1 / 3$ ) one has to perform at least $2 \mathrm{~V}$-cycles between every $\lambda$-FMG step.

A crucial feature in any numerical approach is a reliable estimate of the discretization errors. In our case we estimate the error by measuring the difference in total energy between two consecutive $\lambda$-FMG steps. Assuming that the error drops by a constant factor, $\sigma$, during every $\lambda$-FMG step (see Section 2.5), one can estimate the discretization error to be

$$
\mathscr{E}_{f} \approx\left|\left(f^{(i)}-f^{(i-1)}\right) /\left(\sigma_{f}^{-1}-1\right)\right|
$$

where $f^{(i)}$ is the value of a global function, e.g. the total energy, and $\sigma_{f}$ is the factor by which the discretization error drops in every $\lambda$-FMG step. The $(i)$ superscript denotes here and below a quantity which is evaluated at the end of the $i^{\text {th }} \lambda$-FMG step. In the case where $f=E_{K S}$ we expect that $\sigma_{E_{K S}}=\sigma$, where $\sigma$ is the factor used in the definition of the $\lambda$-FMG cycle. For other quantities, $\sigma_{f}$ can be estimated as

$$
\sigma_{f} \approx\left|\left(f^{(i)}-f^{(i-1)}\right) /\left(f^{(i-1)}-f^{(i-2)}\right)\right|
$$

We tested this estimator for $f=E_{K S}$ in the case of the $\mathrm{H}_{2}$ molecule with the $4^{\text {th }}$-order Laplace discretization. The results are shown in Figure 6, compared to the true error. The latter is defined as the difference between the current value of the total energy and its value in the final $\lambda$-FMG step, which has been confirmed to be the same to six significant digits as the results obtained by Makmal et al. ${ }^{17}$

Assuming that the refinement algorithm works properly, the $\lambda$-FMG cycle is supposed to yield an asymptotic accuracy-to-work ratio of a regular problem without local refinement, $\mathscr{E} \sim \mathscr{W}^{-4 / 3}$, as discussed above Eq. (35). The exponents measured for the various molecules are shown in Table Table 1 and are indeed distributed around to this theoretical value. 


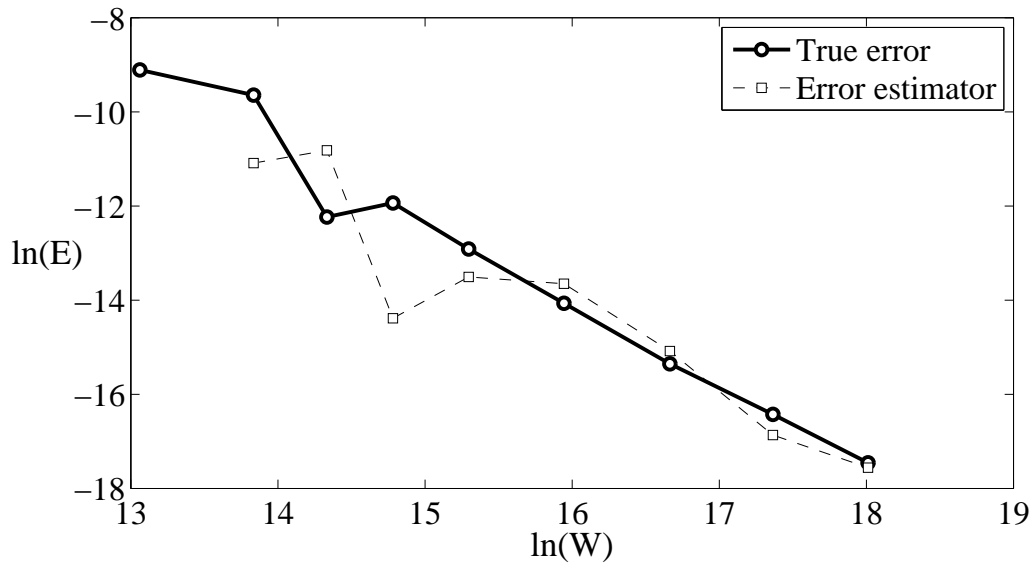

Figure 6: Analysis of the error in the total energy of the $\mathrm{H}_{2}$ molecule during the $\lambda$-FMG cycle. The total work, $W$, is assumed to be $W \propto \sum L_{\text {total }}^{i}$, where $L_{\text {total }}^{i}$ denotes the total number of grid points used in $V$-cycle $i$ and the sum is over the current and previous $\mathrm{V}$-cycles. The figure shows the true error in the total energy, which is the difference between the total energy in the current $\lambda$-FMG step and the value in the final $\lambda$-FMG step (not shown). This is compared with the error estimate obtained from Eq. (39).

Table 1: Definition of the various molecular systems tested in the present work. Left column: Definition of geometrical parameters (distances are given in Bohr). Middle column: asymptotic accuracy-to-work ratio observed during the $\lambda$-FMG cycle. Right column: run times required for obtaining an accuracy of $10^{-4}$ Hartree on a single processor, in minutes.

\begin{tabular}{|c|c|c|c|}
\hline Mol. & Geometry & $\mathscr{E} \propto \mathscr{W}^{-\gamma}, \gamma=$ & Run Time \\
\hline $\mathrm{H}_{2}$ & $\mathrm{R}_{H-H}=1.446$ & 1.35 & 6.4 \\
\hline $\mathrm{Li}_{2}$ & $\mathrm{R}_{L i-L i}=5.120$ & 1.41 & 23 \\
\hline $\mathrm{BH}$ & $\mathrm{R}_{B-H}=2.373$ & 1.48 & 16 \\
\hline $\mathrm{N}_{2}$ & $\mathrm{R}_{N-N}=2.068$ & 1.34 & 20 \\
\hline $\mathrm{H}_{2} \mathrm{O}$ & $\begin{array}{c}\mathrm{R}_{O-H}=1.8336 \\
\alpha=104.6898^{\circ}\end{array}$ & 1.43 & 22 \\
\hline $\mathrm{CO}_{2}$ & $\mathrm{R}_{C-O}=2.19624$ & 1.42 & 49 \\
\hline $\mathrm{C}_{6} \mathrm{H}_{6}$ & $\begin{array}{l}\mathrm{R}_{C-C}=2.6200 \\
\mathrm{R}_{C-H}=2.0684\end{array}$ & 1.52 & 353 \\
\hline $\mathrm{CH}_{3} \mathrm{COOH}$ & See Ref. 49 & 1.36 & 73 \\
\hline
\end{tabular}


Table Table 2 shows the total energies and the highest occupied eigenvalues calculated using CARMA for several molecules. The results are compared to those obtained for the same geometries using the Gaussian program ${ }^{50}$ with two relatively large basis-sets, aug-cc-pVTZ ${ }^{51,52}$ and aug-pc4, ${ }^{53}$ and another standard basis-set, 6-31G(d,p). ${ }^{54,55}$ Results for the diatomic molecules are additionally compared to those obtained from an all-electron fully numerical solution based on a prolate-spheroidal grid. ${ }^{16,17}$ The comparison with the results obtained on the prolate-spheroidal grid shows complete agreement to all significant digits, thus confirming the validity of our numerical approach. Comparison with the results of the largest basis set, aug-pc4, reveals residual differences of the order of $10^{-6}$ Hartree for diatomic molecules and $10^{-5}$ Hartree for larger molecules such as $\mathrm{C}_{6} \mathrm{H}_{6}$. The difference between the CARMA and basis sets results, normalized per electron, is approximately constant $\left(3 \cdot 10^{-6}\right.$ for aug-pc $4,7 \cdot 10^{-4}$ for aug-cc-pVTZ and $4 \cdot 10^{-3}$ for 6-31G(d,p)), indicating that the source of the error is the remaining inaccuracy in the treatment of the core region by the basis set. This comparison demonstrates that our approach can yield high accuracy results for a variety of many-electrons systems, including molecules with a nontrivial geometry such as $\mathrm{C}_{6} \mathrm{H}_{6}$ and $\mathrm{CH}_{3} \mathrm{COOH}$. It can thus be used as an independent, high-accuracy benchmark through which one can gauge the quality of basis sets and other approximations.

\section{Conclusions}

We presented a method for solving the all-electron Kohn-Sham equation in molecules, using an approximation-free discretization based on a locally-refined multigrid approach. The solution is obtained on a set of Cartesian grids, from coarse ones that span the entire molecule to very fine ones that span only a small volume around each atom. The singularity of the potential requires the use of a relatively large number of small refinement patches around each atom (between 10 to 15 in the above calculations), in comparison to other known application of local-mesh refinement. The precise value of the mesh spacing used in every point in space is determined automatically using the $\lambda$-FMG algorithm, which aims at obtaining a desired accuracy with the minimal amount 
Table 2: Comparison between fully-numerical all-electron calculations, obtained using the present approach (locally-refined multrigrid) and on a prolate-spheroidal grid, and between gaussian basisset calculations. The latter are performed using the Guassian program ${ }^{50}$ based on the aug-pc $4,{ }^{53}$ aug-cc-pVTZ ${ }^{51,52}$ and 6-31G(d,p) ${ }^{54,55}$ basis-sets and an ultra-fine real-space grid (used to compute the $V_{x c}$ ). All results are obtained using the Perdew-Wang parametrization of the LDA exchange correlation functional. ${ }^{39}$ HOMO denotes the eigenvalue of the highest occupied orbital. (a) and (b) superscripts denote results by Makmal et al. ${ }^{17}$ and by Grabo et al., ${ }^{16}$ respectively.

\begin{tabular}{|c|c|c|c|c|c|c|}
\hline & & \multicolumn{2}{|c|}{ All-electron fully numerical } & \multicolumn{3}{|c|}{ Gaussian basis set } \\
\hline & Molecule & multrigrid & prolate-spher. & aug-pc4 & aug-cc-pVTZ & $6-31 G(d, p)$ \\
\hline \multirow{8}{*}{$\begin{array}{c}\text { Total } \\
\text { energy } \\
\text { (Hartree) }\end{array}$} & $\mathrm{H}_{2}$ & $-1.1376920(5)$ & $-1.137692(1)^{a}$ & -1.137690 & -1.13712 & -1.13558 \\
\hline & $\mathrm{Li}_{2}$ & $-14.72446(2)$ & $-14.7244(5)^{a}$ & -14.724440 & -14.72315 & -14.71732 \\
\hline & $\mathrm{BH}$ & $-24.97695(6)$ & $-24.97695(2)^{a}$ & -24.976949 & -24.97496 & -24.96345 \\
\hline & $\mathrm{N}_{2}$ & $-108.6958(6)$ & $-108.6959^{b}$ & -108.69583 & -108.68562 & -108.63563 \\
\hline & $\mathrm{H}_{2} \mathrm{O}$ & $-75.91091(6)$ & & -75.91089 & -75.90293 & -75.85228 \\
\hline & $\mathrm{CO}_{2}$ & $-187.2802(4)$ & & -187.28017 & -187.26131 & -187.17471 \\
\hline & $\mathrm{C}_{6} \mathrm{H}_{6}$ & $-230.1926(1)$ & & -230.19248 & -230.17108 & -230.08835 \\
\hline & $\mathrm{CH}_{3} \mathrm{COOH}$ & $-227.4075(1)$ & & -227.40739 & -227.38483 & -227.28422 \\
\hline \multirow{8}{*}{$\begin{array}{c}\text { HOMO } \\
\text { eig. } \\
\text { (Hartree) }\end{array}$} & $\mathrm{H}_{2}$ & $-0.3730920(7)$ & $-0.3730920^{a}$ & -0.37309 & -0.37289 & -0.37013 \\
\hline & $\mathrm{Li}_{2}$ & $-0.1186583(1)$ & $-0.1186(6)^{a}$ & -0.11861 & -0.11871 & -0.11650 \\
\hline & $\mathrm{BH}$ & $-0.2040752(6)$ & $-0.2040(7)^{a}$ & -0.20407 & -0.20400 & -0.18483 \\
\hline & $\mathrm{N}_{2}$ & $-0.38260(7)$ & $-0.3826^{b}$ & -0.38261 & -0.38289 & -0.36770 \\
\hline & $\mathrm{H}_{2} \mathrm{O}$ & $-0.27078(4)$ & & -0.27078 & -0.27075 & -0.23085 \\
\hline & $\mathrm{CO}_{2}$ & $-0.3423(3)$ & & -0.34241 & -0.34232 & -0.32673 \\
\hline & $\mathrm{C}_{6} \mathrm{H}_{6}$ & $-0.2412(2)$ & & -0.24127 & -0.24115 & -0.22654 \\
\hline & $\mathrm{CH}_{3} \mathrm{COOH}$ & $-0.24564(1)$ & & -0.24572 & -0.24562 & -0.22503 \\
\hline
\end{tabular}


of computational work. This algorithm allows us to solve the Kohn-Sham equation with an accuracy to work tradeoff which is similar to that of obtained in simple discretizations of nonsingular problems.

While the standard multigrid approach is often demonstrated for linear and regular equations, here the combination of a nonlinear eigenproblem and a singular potential requires advanced multigrid techniques, described in detailed in Section 2. Specifically, in order to accelerate the convergence of the residuals of the nonlinear set of equations, their self-consistent solution is obtained using a one-shot V-cycle, whereby the whole set is solved on each grid level during the V-cycle. The boundaries between the large number of refinement patches yield a significantly asymmetric Laplace discretization, which may destabilize this self-consistency cycle. To overcome this problem we introduce a modified Rayleigh-Ritz procedure. This procedure may be necessary in the future to accelerate the self-consistency cycle and achieve a linear scaling of the computational power with the number of atoms in the molecule.

The results obtained using the above approach agree to all significant digits with previous all-electron calculation of diatomic molecules, where the Kohn-Sham equation was solved on a prolate-spheroidal grid. ${ }^{16,17}$ For more complex molecules, namely $\mathrm{H}_{2} \mathrm{O}, \mathrm{CO}_{2}, \mathrm{CH}_{3} \mathrm{COOH}$ and $\mathrm{C}_{6} \mathrm{H}_{6}$, our results show very good agreement with gaussian basis set calculations, up to a level which appears to correspond to the accuracy limit of the basis set approximation. For the purposes of this article, all results were obtained using a simple exchange-correlation energy functional, namely the LDA one, with a fixed geometry of the molecule. Nevertheless, the above approach is readily applicable to more advanced physical scenarios. In the next and final section, we point out various avenues for further improvement and refinement of our approach. In particular, we describe a slow convergence phenomenon of the residuals of Kohn-Sham equation for relatively high eigenvalues and suggest that it may be removed using inter-grid interpolation operators that are adapted to the oscillatory form of the wavevectors. 


\section{Future perspective}

In this section, we discuss possible extensions of the above approach. The interested reader may also wish to consider a general survey of research directions in multigrid implementations of DFT, found in section 9 of Brandt. ${ }^{42}$

Our approach is readily applicable to more elaborate physical scenarios, one of which is the use of orbital-dependent exchange-correlation energy functionals. ${ }^{7}$ The use of such functionals within the Kohn-Sham scheme requires the additional solution of an integro-differential equation known as the optimized effective potential (OEP) equation. ${ }^{7}$ In the OEP approach, the self-consistent calculation of the exchange-correlation potential consumes a significant portion of the computational work. As demonstrated above for the case of the Hartree potential, using the one-shot V-cycle to compute this exchange-correlation potential may accelerate its convergence in comparison to similar non-multigrid implementations.

Other interesting physical features that can be incorporated in this approach are geometrical optimization and periodic boundary conditions. The $\lambda$-FMG cycle provides a natural framework for an efficient geometrical optimization process, whereby the coarse relocations of the atoms are performed during the initial stages of the cycle on a set of coarse grids, during which the calculation of the forces is less demanding. The grid structure implemented in this study also provides an efficient way of modifying the wavevectors upon a relocation of an atom. The small refinement grids should move with the atom they enclose, requiring little change to their wavevectors. On the other hand, the coarse grids that remain static may require several additional relaxation steps or a $W$-cycle ${ }^{20}$ to account for the change in the geometry of the molecule. Because the grid points in the finest 'static' grid level and the coarsest 'movable' grid level may not overlap exactly, this would require employing grid transfer operators between two nonoverlapping grid levels.

In the present implementation of the above approach, all the wavevectors are represented on all the grid levels. Our algorithm can be made more efficient by not representing wavevectors that are localized around certain atoms on local-refinement grids that are spanned around other atoms. As a result, the necessary computational work and memory storage would scale linearly with the 
number of electrons in the molecule, rather than as the number of electrons times the number of atoms. The efficiency can be further increased by keeping fine-to-coarse defect corrections of very fine grid levels frozen during some of the V-cycles, as suggested in section 15 in Brandt. ${ }^{20}$ Further improvement in efficiency can be obtained by allowing refinement grids to overlap. In CARMA, when the $\lambda$-FMG algorithm causes two refinement grids to expand into the same region they are united into a single larger refinement grid, as demonstrated in Figure 4. Allowing refinement grids to overlap can reduce the number of grid points required for solving complex molecules such as $\mathrm{C}_{6} \mathrm{H}_{6}$. Such overlap may also be necessary in order to obtain optimal parallelization of the algorithm.

One possible extension of the mixing scheme discussed in Section 2.4, which was crucial for the convergence of the one-shot V-cycle, would be to mix between the approximation vectors in previous $V$-cycles and to incorporate this type of mixing also in the Rayleigh-Ritz procedure. Another possible approach to this issue is to perform a simultaneous relaxation of Eqs. (17), (18),(20), and (21) instead of the usual Kaczmarz relaxation. This idea is known in the multigrid methodology as collective relaxation (see section 3.4 in $\mathrm{Brandt}^{20}$ ). In order to implement it, one would have to expand the set of equations to linear order in terms of small changes in $\left\{\varphi_{k}\right\}, V_{H}$ and $V_{x c}$. Each collective Kaczmarz iteration should then consist of diagonalizing the resulting matrix and altering together all the occupied wavevectors, $V_{H}$, and $V_{x c}$, at a certain grid point and at its nearest neighbours.

In Appendix A we discuss several possible extensions of the Constrained Asymmetric Rayleigh Ritz (CARR) process, introduced in Section 2.7, that may increase the efficiency of the $V$-cycle. One possible direction is to perform the CARR procedure on coarse grids. Another idea is to accelerate the convergence of the self-consistency cycle, by taking into account small changes in the potential while computing the optimal recombination of the wavevectors. In addition, one may consider recombining the wavevectors with their values in previous V-cycles. This amounts to using the multigrid cycle as a preconditionner for the conjugate gradient algorithm. This idea has yielded significant increase in the efficiency of various multigrid implementations in other fields, 
as discussed in section 7.3 of Brandt. ${ }^{20}$

The approach presented above did not attain the textbook $V$-cycle convergence rates. During a single 'healthy' V-cycle the residuals of the equations that being solved should drop by a factor of about $10 .^{20}$ The typical factors found using CARMA varied between 3 to 6 in simple systems such as $\mathrm{H}_{2}$ or $\mathrm{Li}_{2}$. Oscillatory wavevectors appeared to converge more slowly than the smooth ones, causing many-electron systems such as $\mathrm{CO}_{2}$ or $\mathrm{C}_{6} \mathrm{H}_{6}$, where such oscillatory wavevectors are occupied, to converge even more slowly at a rate of about 2. Because we have observed this issue also in the linear case (in high eigenvectors of the hydrogen atom, where $V_{\text {ion }}=1 / r$ and $V_{x c}=V_{H}=0$ ), we may exclude nonlinear effects as a possible explanation for this phenomenon. In addition, the absence of this issue in the harmonic case $\left(V_{i o n}=r^{2}\right.$ and $\left.V_{x c}=V_{H}=0\right)$, suggests that the polynomial based grid transfer operators might not provide a good coarse description of the oscillatory wavevectors in the proximity of the cores. If this is indeed the source of the problem, it may be solved by constructing optimal interpolation operators, following the approach of the exact interpolation scheme (EIS). ${ }^{56,57}$ Another possible direction is to use local refinement grids that are based on spherical coordinates, where the angular behavior of the wavevectors in the vicinity of the cores is regular. The EIS should also be more efficient on spherical refinement grids, where one should only fit the radial dependence of the interpolation operators to the structure of the wavevectors.

\section{Acknowledgement}

We thank A. Biller, A. Makmal, A. Natan, and L. Segev for helpful discussions. We further thank J. M. L. Martin for expert advice on gaussian basis-set issues. This work was supported by the European Research Council and the Lise Meitner Minerva Center for Computational Chemistry. 


\section{A Constrained Asymmetric Rayleigh Ritz (CARR) process}

In this appendix, we present a modified Rayleigh-Ritz procedure, which we name the Constrained Asymmetric Rayleigh Ritz (CARR) process. The original purpose of the CARR process is to find an optimal recombination of the wavevectors at the end of each $V$-cycle, that would minimize the residual of the Kohn-Sham equation, per each wavevector, while maintaining orthogonality between degenerate wavevectors. The CARR process allows the one-shot $V$-cycle, presented in Section 2.4 , to converge smoothly to very low residuals, well below the level of discretization errors. Such convergence, although not necessary for obtaining additional physical precision, was needed in order to verify that all the components of the solver function properly. In addition, the CARR process has several advantages, discussed below, which may be crucial for studying larger molecular systems and for using orbital-dependent exchange-correlation energy functionals.

The starting point of the CARR algorithm is the original set of wavevector, $\left\{\varphi_{k}\right\}$, and eigenvalues, $\left\{\varepsilon_{k}\right\}$, where $k=1, \ldots, N$. The algorithm iteratively seeks a set of new eigenvalues, $\left\{\varepsilon_{k}^{\prime}\right\}$, and a recombination matrix, $b_{k j}$, which defines a new set of wavevectors via $\varphi_{k}^{\prime}=\sum_{j=1}^{N} b_{k j} \varphi_{j}$. Each wavevector, $\left\{\varphi_{k}^{\prime}\right\}$, should minimize the residual of its Kohn-Sham equation while remaining normalized and orthogonal to eigenvectors with a similar eigenvalue. These conditions can be translated to the following set of equations over $\mathbf{b}_{k}$ and $\varepsilon_{k}^{\prime}$ :

$$
M_{k} \mathbf{b}_{k}=\varepsilon_{k} \mathbf{b}_{k} \quad \mathbf{b}_{k}^{T} U \mathbf{b}_{l}=\delta_{k l} \quad \forall l:\left|\varepsilon_{k}-\varepsilon_{l}\right| \leq \Delta_{\varepsilon}
$$

where $\Delta_{\varepsilon}$ is an energy cutoff, whose value is discussed below, and

$$
\left(M_{k}\right)_{i j} \equiv\left\langle\varphi_{i}^{h}, H_{k}^{h} \varphi_{j}^{h}\right\rangle, \quad U_{i j} \equiv\left\langle\varphi_{i}^{h}, \varphi_{j}^{h}\right\rangle
$$

The above inner products are evaluated throughout space on the finest available grid using the integration weights described in Section 2.2. The $k$ subscript of $M$ and $H$ imply that the Hamiltonian may in fact be discretized differently for each wavevector or contain an orbital-dependent poten- 
tial, as found in some advance approximations of $V_{x c}{ }^{7}$ Allowing for such orbital-dependence is an important advantage of the CARR process over the standard Rayleight-Ritz process.

Equation (41) can be linearized by assuming small changes in $\mathbf{b}_{k}$ and in $\varepsilon_{k}$, which yields an iterative solution given by

$$
\begin{gathered}
\left(M_{k}-\varepsilon_{k}^{(t)} I\right) \mathbf{b}_{k}^{(t+1)}-\mathbf{b}_{k}^{(t)} \varepsilon_{k}^{(t+1)}=-\varepsilon_{k}^{(t)} \mathbf{b}_{k}^{(t)} \\
\left(1+\delta_{k l}\right) \mathbf{b}_{k}^{(t+1), T} U \mathbf{b}_{l}^{(t)}=\delta_{k l}+\mathbf{b}_{k}^{(t), T} U \mathbf{b}_{l}^{(t)}
\end{gathered}
$$

Here $\mathbf{b}_{k}^{(t)}, \varepsilon_{k}^{(t)}$ denote the approximation of the eigenvector and eigenvalue at the $t^{\prime}$ th iteration, respectively. The number of eigenvalues obeying $\left|\varepsilon_{k}-\varepsilon_{l}\right| \leq \Delta_{\varepsilon}$ is denoted, here on, as $N_{\Delta}^{k}$, and their indices are given as $\left(k, i_{1}, \ldots, i_{N_{\Delta}^{k}-1}\right)$. In this notation, Eq. (43) can be written as a set of $N+N_{\Delta}^{k}$ linear equations given by

$$
\left(\begin{array}{cccc}
\varepsilon_{k}^{(t)} I-M_{k} & \mathbf{b}_{k}^{(t)} & \mathbf{b}_{i_{1}}^{(t)} & \cdots \\
2 \mathbf{b}_{k}^{(t), T} U & 0 & 0 & \\
\mathbf{b}_{i_{1}}^{(t), T} U & 0 & 0 & \\
\vdots & & & \ddots \\
\mathbf{b}_{i_{\Delta}^{k}-1}^{(t), T} U & 0 & 0 &
\end{array}\right)\left(\begin{array}{c}
\mathbf{b}_{k}^{(t+1)} U \\
\varepsilon_{k}^{(t+1)} \\
\mu_{1} \\
\vdots \\
\mu_{i_{\Delta}^{k}-1}
\end{array}\right)=\left(\begin{array}{c}
\varepsilon_{k}^{(t)} \mathbf{b}_{k}^{(t)} \\
1+\left|\mathbf{b}_{k}^{(t)} U \mathbf{b}_{k}^{(t)}\right| \\
0 \\
\vdots \\
0
\end{array}\right)
$$

where $\mu$ is a vector of ghost parameters. One drawback of this approach is that, unlike the standard Rayleigh-Ritz process, it relies on an initial guess for the eigenvectors and eigenvalues. A natural choice which often works is to use the identity matrix, $b^{(0)}=I$, and set also $\varepsilon_{i}^{(0)}=\varepsilon_{i}$. However, during the continuation process, where the wavevectors and potentials may undergo significant changes between every $V$-cycle, it is necessary to start the process by diagonalizing first the symmetrized Ritz matrix, $\frac{1}{2}\left(M+M^{T}\right)$. The diagonalization yields a diagonal matrix, $D$, and a transformation matrix, $P$, such that $D=P^{T} \frac{1}{2}\left(M+M^{T}\right)^{\prime} P$. The starting point of the CARR process should in this case be $b^{(0)}=P$ and $\varepsilon_{i}^{(0)}=D_{i i}$.

The value of $\Delta_{\varepsilon}$ has to be chosen carefully in order to suit the current level of discretization 
error. When $\Delta_{\varepsilon}$ is taken to be too large, the CARR process cannot separate vector components with different eigenvalues as needed for minimizing the residuals (see explanation below Eq. (10)), leading in turn to very slow convergence rates. On the other hand, when $\Delta_{\varepsilon}$ is too small the CARR process may yield the same transformation matrix, $b$, as the one obtained using by the standard Rayleigh-Ritz procedure. In this case, as discussed in Section 2.7, the residuals cannot converge below the level of discretization errors.

In general the CARR process converges very quickly, and in most cases it reaches its fixed point after no more than 5 iterations. We found, however, that the process converges very slowly, or even diverges, if it involves wavevectors with positive eigenvalues. These wavevectors are not localized around the atom and their convergence is not important for the calculation of steady-state electronic structure. In order to avoid the convergence problem we simply excluded them from the CARR process.

One may consider several extensions to the CARR process in order to improve the efficiency of the one-shot $V$-cycle. One possible extension is to perform the CARR procedure not only on the finest grid level, but on several coarse grid levels. This would require transferring fine corrections to the $M_{k}$ and $U$ matrices, defined in Eq. (41), using Eq. (25), as well as using the right hand side of the coarse Kohn-Sham equations.

The standard Rayleigh-Ritz process, as well as the CARR process described above, require integrating $N^{2}$ inner products over space. For complex molecules which consist of many eigenvectors, this integration requires a significant amount of computational work. In order to increase the efficiency of this evaluation, the wavevectors should be separated into partially-overlapping subspaces, each of which includes wavevectors with a common physical property such as being located around a certain atom. The CARR process should then be performed within each subspace, while alternating between between the subspaces. The computer power required for such an implementation would scale as $O(N)$, instead of $O\left(N^{2}\right)$.

Another possible extension of the CARR process is to take into account small changes in the Hartree and exchange-correlation potentials when seeking for the optimal recombination of the 
wavevector. In order to formulate the resulting procedure, we first examine the change in the electron density due to a small change in the eigenvector $k$, which is given by

$$
\delta \rho^{(k)}(x)=\sum_{i=1}^{N}\left(1+\delta_{k i}\right)\left(b_{k, i}-\delta_{k i}\right) f_{k} \varphi_{i}(x) \varphi_{k}(x)
$$

where $f_{k}$ is the orbital occupation-number. The resulting change in the potentials is given by

$$
\begin{aligned}
& \delta V_{x c}^{(k)}(x)=\left.\frac{\delta V_{x c}}{\delta \rho}\right|_{\rho(x)} \delta \rho^{(k)}(x) \\
& \delta V_{H}^{(k)}(x)=\sum_{i=1}^{N} f_{k}\left(1+\delta_{k i}\right)\left(b_{k, i}-\delta_{k i}\right) b_{k, i} U_{k, i}(x),
\end{aligned}
$$

where $U_{k, i}$ are the solutions of the Poisson equation

$$
\nabla^{2} U_{k, i}(x)=-4 \pi \varphi_{k}(x) \varphi_{i}(x)
$$

These terms can then be incorporated in the iterative form of Eq. (43), yielding

$$
\left(M_{k}+\delta M_{k}^{(1)}-\varepsilon_{k}^{(t)} I\right) \mathbf{b}_{k}^{(t+1)}-\mathbf{b}_{k}^{(t)} \varepsilon_{k}^{(t+1)}=\left(\delta M_{k}^{(2)}-\varepsilon_{k}^{(t)}\right) \mathbf{b}_{k}^{(t)}
$$

where the addition to matrix in the LHS is given by

$$
\left(\delta M_{k}^{(1)}\right)_{i j}=2 \int d x \varphi_{i}(x)\left[\sum_{l, m}\left(b_{l, m}^{(t)}-\delta_{l m}\right) f_{m} R_{l m}(x) \varphi_{j}(x)+\sum_{m} f_{k} b_{k, m}^{(t)} \varphi_{m}(x) R_{k j}(x)\right]
$$

with

$$
R_{l m}(x)=\left.\frac{\delta V_{x c}}{\delta \rho}\right|_{\rho(x)} \varphi_{l}(x) \varphi_{m}(x)+U_{l, m}(x) .
$$

The additional matrix in the RHS of Eq. (50) is given by

$$
\left(\delta M_{k}^{(2)}\right)_{i j}=2 \int d x \varphi_{i}(x) \varphi_{j}(x) f_{k} R_{k k}(x)
$$


Here the sum over $m$ and $l$ runs from 1 to $N$ and the integral over $x$ is evaluated using the fine integration scheme, presented in Section 2.2.

Performing this kind of recombination should in principle add a significant computational cost to the one-shot V-cycle, especially due to the solution of the $N^{2}$ Poisson equation defined in Eq. (49). However, because $U_{k, i}$ are relatively smooth (similarly to the Hartree potential), they need be computed only on relatively coarse grid levels. In this way, the work invested in the solution of Eq. (49) would be comparable to that invested in the computation of the Hartree potential over the entire grid formation. This approach may also useful for the evaluation of certain orbital-dependent exchange-correlations functional that depend on $U_{k, i}$ as well.

\section{References}

(1) Parr, R. G.; Yang, W. Density-functional theory of atoms and molecules; Oxford university press, 1989; Vol. 16.

(2) Gross, E. K.; Dreizler, R. M. Density functional theory; Plenum Publishing Corporation, 1995; Vol. 337.

(3) Pickett, W. E. Comput. Phys. Rep. 1989, 9, 115 - 197.

(4) Chelikowsky, J. R.; Cohen, M. L. Handbook on Semiconductors 1992, 1, 59.

(5) Cramer, C. J. Essentials Of Computational Chemistry; John Wiley and Sons, New York, 2002; p 166.

(6) Hirata, S.; Ivanov, S.; Grabowski, I.; Bartlett, R.; Burke, K.; Talman, J. D. J. Chem. Phys. 2001, $115,1635$.

(7) Kümmel, S.; Kronik, L. Rev. Mod. Phys. 2008, 80, 3.

(8) Gómez-Abalo, R.; Li, X.; Scheffler, M.; Ambrosch-Draxl, C. Phys. Rev. Lett. 2008, 101, 106404. 
(9) Pask, J.; Sterne, P. Modelling and Simulation in Materials Science and Engineering 2005, 13, R71.

(10) Fattebert, J. L.; Hornung, R. D.; Wissink, A. M. J. Com. Phys. 2007, 223, 759-773.

(11) Bylaska, E. J.; Holst, M.; Weare, J. H. J. Chem. Theory Comput. 2009, 5, 937-948.

(12) Fang, J.; Gao, X.; Zhou, A. J. Comput. Phys. 2012, 231, 3166-3180.

(13) Bao, G.; Hu, G.; Liu, D. J. Comput. Phys. 2012.

(14) Lehtovaara, L.; Havu, V.; Puska, M. The Journal of chemical physics 2009, 131, 054103.

(15) Schauer, V.; Linder, C. J. Comput. Phys. 2013.

(16) Grabo, T.; Kreibich, T.; Gross, E. K. U. Molecular Engineering 1997, 7, 27-50.

(17) Makmal, A.; Kümmel, S.; Kronik, L. J. Chem. Theory Comput. 2009, 5, 1731-1740.

(18) Modine, N. A.; Zumbach, G.; Kaxiras, E. Phys. Rev. B 1997, 55, 10289.

(19) Beck, T. L. Rev. Mod. Phys. 2000, 72, 1041-1080.

(20) Brandt, A.; Livne, O. E. Multigrid Techniques - 1984 Guide with Applications to Fluid Dynamics (Revised Edition); SIAM, Philadelphia, PA, 2011.

(21) Briggs, W. L.; Emden, H. V.; McCormick, S. F. A Multigrid Tutorial, 2nd ed.; SIAM, Philadelphia, PA, 2000.

(22) Trottenberg, U.; Oosterlee, C.; Schuller, A. Multigrid; Academic Press, New York, 2000.

(23) Brandt, A. Mathematics of Computation, American Mathematical Society 1977, 31, 333-390.

(24) Frehse, J.; Pallaschke, D.; Trottenberg, U. Special Topics in Applied Mathematics 1980, 31, 91-128. 
(25) Kohn, S.; Weare, J.; Ong, E.; Baden, S. 8. SIAM conference on parallel processing for scientific computing; 1996.

(26) Venkatesh, P. K. Physica B 2001, 318, 121-139.

(27) Saarikoski, H.; Räsänen, E.; Siljamäki, S.; Harju, A.; Puska, M.; Nieminen, R. The European Physical Journal B-Condensed Matter and Complex Systems 2002, 26, 241-252.

(28) Feng, G.; Beck, T. L. Phys. Stat. Sol. (b) 2006, 243, 1054-1062.

(29) Wijesekera, N. R.; Feng, G.; Beck, T. L. Phys. Rev. B 2007, 75, 115101.

(30) Bernholc, J.; Hodak, M.; Lu, W. J. Phys.: Condens. Matter 2008, 20, 294205.

(31) Shimojo, F.; Kalia, R. K.; Nakano, A.; Vashishta, P. Phys. Rev. B 2008, 77, 085103.

(32) Beck, T. L. Intern. J. of Quan. Chem. 1998, 65, 477-486.

(33) Forenbrg, B.; Sloan, D. M. Acta Numerica 1994, 3, 203-267.

(34) Brandt, A. Applied Mathematics and Computation 1986, 19, 23-56.

(35) Brandt, A.; McCormick, S.; Ruge, J. SIAM J. Sci. Stat. Comput. 1983, 4, 244-260.

(36) Kaczmarz, S. Bulletin International de l'Academie Polonaise des Sciences et des Lettres 1937, A 35, 335-337.

(37) Bai, D.; Brandt, A. SIAM J. Sci. Stat. Comput. 1987, 8, 109-134.

(38) Perdew, J.; Zunger, A. Phys. Rev. B 1981, 23, 5048-5079.

(39) Perdew, J. P.; Wang, Y. Phys. Rev. B 1992, 45, 13244, In Gaussian this parametrization is used by adding the keyword "SPW91 IOp(3/78=0000010000)".

(40) Landau, L. D.; Lifshitz, E. M. The Classical Theory of Fields, 4th ed.; Pergamon, Oxford, 1975; pp 97-99. 
(41) Burdick, W. R.; Saad, Y.; Kronik, L.; Vasiliev, I.; Jain, M.; Chelikowsky, J. R. Computer Physics Communications 2003, 156, 22 - 42.

(42) Brandt, A. In Multiscale Scientific Computation: Review 2001; T Barth, R. H., Chan, T., Eds.; Springer Verlag, 2001.

(43) Eyert, V. J. Compu. Phys. 1996, 124, 271-285.

(44) Jain, M.; Kronik, L.; Chelikowsky, J. R.; Godlevsky, V. V. Phys. Rev. B 2001, 64, 245205.

(45) Remediakis, I. N.; Kaxiras, E.; Chen, M.; Friend, C. M. J. Chem. Phys. 2003, 118, 6046.

(46) Kohn, S.; Ong, J. H. W. E. M. G.; Baden, S. B. In Software and computational issues in parallel structured adaptive mesh methods for electronic structure.

(47) Griebel, M.; Zumbusch, G. In Hash Based Adaptive Parallel Multilevel Methods with SpaceFilling Curves; Rollnik, H., Wolf, D., Eds.; John von Neumann Institut für Computing, Forschungszentrum Jülich Germany, 2002; Vol. 9, pp 479-492.

(48) Ewing, R. E.; Lazarov, R. D.; Vassilevski, A. T. SIAM J. on Numer. Anal. 1990, 31, 16051622.

(49) Nuclei coordinates in Bohr radii: $\mathrm{C}(0,0,0), \mathrm{O}(0,0,1.207217), \mathrm{O}(1.14105,0,-0.716555), \mathrm{C}(-$ $1.198642,-0.001044,-0.875179)$, H(1.869007,0.000356,-0.056682), H(-1.184665,0.882417,1.532696), H(-1.183545,-0.884834,-1.532234), H(-2.106195,-0.001464,-0.260919).

(50) Frisch, M. J. et al. Gaussian 03; Gaussian, Inc.: Wallingford, CT, 2004.

(51) Kendall, R. A.; Dunning Jr, T. H.; Harrison, R. J. J. Chem. Phys. 1992, 96, 6796.

(52) Woon, D. E.; Dunning Jr, T. H. J. Chem. Phys. 1993, 98, 1358.

(53) Jensen, F. Theor. Chem. Acc. 2005, 113, 187-190.

(54) Hehre, W. J.; Ditchfield, R.; Pople, J. A. J. Chem. Phys. 1972, 56, 2257. 
(55) Francl, M. M.; Pietro, W. J.; Hehre, W. J.; Binkley, J. S.; Gordon, M. S.; DeFrees, D. J.; Pople, J. A. J. Chem. Phys. 1982, 77, 3654-3665.

(56) Kushnir, D.; Galun, M.; Brandt, A. IEEE Trans. Pattern Anal. Mach. Intell. 2010, 32, 1377.

(57) Brandt, A.; Brannick, J.; Kahl, K.; Livshits, I. SIAM J. Sci. Comput. 2011, 33, 612-632. 